\title{
Hydrodynamic Simulations of Classical Novae: Accretion onto CO White Dwarfs as SN la Progenitors
}

\section{S. Starrfield ${ }^{* \dagger a}$, M. Bose ${ }^{a}$, C. Iliadis ${ }^{b}$, W. R. Hix ${ }^{c, d}$, C. E. Woodward ${ }^{e}$, R. M. Wagner ${ }^{f, g}$, J. Jóse ${ }^{h}, \mathbf{M}$. Hernanz ${ }^{i}$}

${ }^{a}$ Arizona State University- Earth and Space Exploration, Tempe, AZ,USA; ${ }^{b}$ University of North Carolina - Physics and Astronomy, Chapel Hill, NC; ${ }^{c}$ Oak Ridge National Laboratory, Oak Ridge, TN; ${ }^{d}$ University of Tennessee, Knoxville, TN; ${ }^{e}$ University of Minnesota, Minneapolis, $M N ;{ }^{f}$ Large Binocular Telescope Observatory, Tucson, AZ; ${ }^{g}$ Ohio State University, Department of Astronomy, Columbus, OH; ${ }^{h}$ University Polytechnic of Catalunya, Barcelona, Catalunya, Spain; ${ }^{i}$ Institute of Space Sciences, Barcelona, Catalunya, Spain

E-mail:

starrfieldaasu. edu, mbose2 . asulgmail.com, iliadisephysics.unc.edu, raph@ornl.gov, chickw024@gmail.com, rmw@lbto.org, jordi.jose@upc.edu, hernanz@ieec.uab.es

We report on our continuing studies of Classical Nova explosions by following the evolution of thermonuclear runaways (TNRs) on carbon-oxygen (CO) white dwarfs (WDs). We have varied both the mass of the WD and the composition of the accreted material. Rather than assuming that the material has mixed from the beginning, we now rely on the results of the multidimensional (multi-D) studies of mixing as a consequence of the TNRs in WDs that accreted only Solar matter. The multi-D studies find that mixing with the core occurs after the TNR is well underway and reach enrichment levels in agreement with observations of the ejecta abundances. We report on 3 studies in this paper. First, simulations in which we accrete only Solar matter with NOVA (our 1-D, fully implicit, hydro code). Second, we use MESA for similar studies in which we accrete only Solar material and compare the results. Third, we accrete Solar matter until the TNR is initiated and then switch the composition in the accreted layers to a mixed composition: either $25 \%$ core and $75 \%$ Solar or $50 \%$ core and $50 \%$ Solar. The amount of accreted material is inversely proportional to the initial ${ }^{12} \mathrm{C}$ abundance. Thus, accreting Solar material results in more material to fuel the outburst - much larger than in the earlier studies where mixed materials were used from the beginning. We tabulate the amount of ejected gases, their velocities, and abundances. We predict the amount of ${ }^{7} \mathrm{Li}$ and ${ }^{7} \mathrm{Be}$ produced and ejected by the explosion and compare our predictions to our Large Binocular Telescope (LBT) high dispersion spectra which determined the abundance of ${ }^{7} \mathrm{Li}$ in nova V5668 Sgr. Finally, many of these simulations eject significantly less mass than accreted and, therefore, the WD is growing in mass toward the Chandrasekhar Limit.

The Golden Age of Cataclysmic Variables and Related Objects IV

11-16 September, 2017

Palermo, Italy

\footnotetext{
* Speaker.

${ }^{\dagger}$ This work supported by NSF and NASA grants to ASU
} 


\section{Introduction}

Supernovae of Type Ia (SNe Ia) are typically those supernovae in which neither hydrogen nor helium is seen in any of the spectra obtained during the outburst. However, as the sample size of SN surveys increase, there are events that otherwise would be classified as SN Ia's that in fact do exhibit $\mathrm{H}$ in their spectra (e.g., 1;2). SN Ia have light curves that can be calibrated (3), making them excellent standardizable distance indicators to $z>1(4 ; 5)$. Thus, they have become extremely important tools to determine the structure and evolution of the Universe $(6 ; 7$, and references therein). SN Ia are also important because they contribute a major fraction of the iron group elements to the galactic Interstellar Medium (ISM) and, therefore, to our Solar System. In the past few years, a tremendous effort has gone into studies of their observed properties (cf., $8 ; 5 ; 9 ; 10)$. Nevertheless, the progenitor(s) of SN Ia explosions are, as yet, unknown.

(11) proposed that the explosion involved a carbon-oxygen white dwarf (CO WD) which accreted material from a binary companion until its mass approached the Chandrasekhar Limit. A carbon deflagration/detonation then occured and produced a SN Ia explosion $(12 ; 13 ; 14)$. This pathway is designated as the single degenerate (SD) scenario. Alternatively, a second mechanism, designated the double degenerate (DD) scenario, is invoked in which two CO WDs merge or collide (15).

In this paper we concentrate on the SD scenario. Although there is no agreement on the progenitors, likely systems are the Cataclysmic Variables (CV) and Symbiotic Binaries which are close (or not so close) binary star systems which contain both a WD primary and a larger cooler secondary star that typically fills its Roche Lobe. The cooler star is losing mass through the inner Lagrangian point of the binary and a fraction of this material is accreted by the WD. However, the absence of hydrogen and helium in the spectra of a SN Ia may rule out most CV systems since the WDs in these systems are accreting hydrogen- and helium-rich material. If the WD were to explode, then the accreted envelope would be carried along with the supernova ejecta and be seen in the spectrum. Nevertheless, if a sufficient amount of the accreted material remains on the WD during the accretion process and its mass can gradually grow close to the Chandrasekhar Limit, then the explosion should resemble a SN Ia.

The SD scenario is capable of explaining most of the observed properties of the SN Ia explosion via the delayed detonation hypothesis $(16 ; 17 ; 18 ; 19$, and references therein). Reviews of the various proposals for SN Ia progenitors (13), and the implications of their explosions can be found in (14), (6; 7), (20), and (5). Recent reviews of the observations can be found in (9) and (10).

New evidence in favor of the SD scenario comes from observations of SN 2011fe in M101. They imply that the exploding star was likely a CO WD (21) with a companion that was probably on or near the main sequence (22; 23). However, EVLA (24) and optical (23) observations may have ruled out many types of CVs. In addition, HST studies of the spatial region from which SN $2011 \mathrm{fe}$ exploded, suggest that the progenitor had a luminosity less than $\sim 10^{34} \mathrm{erg} \mathrm{s}^{-1}$.

Moreover, (1) claim that PTF $11 \mathrm{kx}$ was a SN Ia that exploded in a symbiotic nova system. In additioin, the "zoo" of SNe Ia types is increasing as surveys find more and more members (e.g., 25). The most recent results $(26 ; 27)$, in the same issue of Nature, both favor and disfavor the SD scenario. Therefore, since the existence of "Super-Chandra" Ia's suggests that DD mergers are required for these extreme explosions, evidently, on balance there are multiple channels that can 
produce SN Ia explosions including the SD channel (28).

Observations of V445 Pup (Nova Pup 2000) strongly suggest that the SD channel must be viable. There were no signs of hydrogen in the spectrum at any time during the outburst, especially just after discovery, but there were strong lines of carbon, helium, and other elements in the early, optically thick, spectra $(29 ; 30 ; 31 ; 32 ; 33 ; 34)$. Unfortunately, no complete abundance analysis of the spectra obtained early in the outburst exist to enable a determination of an upper limit to the amount of hydrogen that could be hidden. Nevertheless, it is probably extremely small. Because V445 Pup was extremely luminous before the outburst, the secondary is thought to be a hydrogen deficient carbon star (34). Since one of the defining characteristics of a SN Ia explosion is the absence of hydrogen or helium in the spectrum at any time during the outburst or decline, the existence of V445 Pup implies that mass transferring binaries exist in which hydrogen is absent at the time of the explosion and most of the helium is converted to carbon during the nova phase of evolution. The latest spectra show that this system is still in outburst and, therefore, it has not been possible to study the underlying system (35).

In the next Section we discuss problems with the SD scenario. We follow that with Sections where we present the 2 codes used in our work (Section 3), the 3 different studies of accretion onto CO WDs (Section 4), discuss our discovery of ${ }^{7} \mathrm{Li}$ in V5668 Sgr and its implications (Section 5), and end with Conclusions in Section 6.

\section{Problems with the Single Degenerate Scenario}

The SD scenario can result in light curves and other properties that resemble those of a SN Ia explosion; there are, however, significant problems with any of the suggestions for what the progenitors might actually be. In fact, while virtually every type of close binary, or not so close binary, involving a WD has been suggested as a progenitor, the major problem is that there is no hydrogen or helium observed in the SN Ia explosion. However, in virtually every observed binary with a WD that is transferring material, the material is hydrogen rich. The presence of hydrogen suggests either that these systems are not SN Ia progenitors or that the hydrogen and helium is lost from the system prior to the SN Ia explosion. Moreover, many of the suggested classes of binaries are losing mass at prodigious rates into the local ISM and that material should be present when the system explodes and then appear in the spectrum at some time after the outburst. In fact, there are a few SNe Ia where there are narrow lines of hydrogen in the spectrum that indicate circumbinary or ISM material (9). In addition, there was sufficient hydrogen in the spectrum of PTF $11 \mathrm{kx}$ that (1) claim that it was a SN Ia that exploded in a Symbiotic Nova system.

A more serious problem is that it is commonly assumed that only a very narrow range in mass accretion rate ( $\dot{\mathrm{M}}$ : in units of $\mathrm{M}_{\odot} \mathrm{yr}^{-1}$ ) allows the mass of the WD to grow as a result of continued accretion. The basis of this assumption is the work of $(36 ; 37)$. A plot of his results can be found as Figure 5 in (38). This plot has 3 regions on it. For the lowest mass accretion rates, at all WD masses, it is predicted that accretion results in hydrogen flashes which are normally expected to resemble those of Classical Novae. Since the results of a large number of observational studies, in combination with the theoretical predictions, of Classical Nova $(\mathrm{CNe})$ ejecta, imply that more mass is ejected than accreted $(39 ; 40)$ accretion at low rates cannot result in the growth of the WD to the Chandrasekhar Limit. However, hydrodynamic calculations of this process show that the accreted 
material must mix with core material, in order to produce a $\mathrm{CN}$ outburst, and that process is not included in the calculations of $(36 ; 37)$.

For the highest mass accretion rates in this plot, the results of $(36 ; 37)$ imply that the radius of the WD rapidly expands to red giant dimensions. The implication here is that this causes accretion to be halted and further evolution must await the collapse of the extended layers.

There is, however, a third regime predicted by $(36 ; 37)$, intermediate between these two, where the material is expected to burn steadily at the rate it is accreted. The central $\dot{\mathrm{M}}$ of this region is nominally $\sim 3 \times 10^{-7} \mathrm{M}_{\odot} \mathrm{yr}^{-1}$ but it does have a slight variation with WD mass. Those systems that are accreting at the steady burning rate are evolving horizontally in this plot towards higher WD mass and, by some unknown mechanism, the mass transfer in the binary system is stuck in this mass accretion range. The implication, therefore, is that only a narrow range of mass accretion rates will result in the growth in mass of the WD. However, the Super Soft Binary X-ray Sources (SSS) in the LMC are accreting at high rates and are predicted to be in the steady burning regime (41). Unfortunately, there are insufficient SSS systems for them to be the only SN Ia progenitors and recent observations of nearby SN Ia explosions appear to rule out luminous progenitors. Moreover, the observations of CVs and other systems with accreting WDs show that they are accreting at rates that are not within the steady burning regime and, therefore, the WD cannot be growing in mass.

However, the calculations reported in $(36 ; 37)$ assume a steady state solution and imply that the only parameters that affect the evolution of an accreting WD are its mass and $\dot{M}$. These calculations do not take into account the chemical composition of the accreting material, the chemical composition of the underlying WD, if mixing of accreted material with core material has taken place, or the thermal structure of the underlying WD. Moreover, the effects of previous (or continuing) outbursts on the thermal and compositional structure of the WD (42) are not considered. It is well known that all these parameters affect the evolution of the WD (43; 44). In the Section 4 we report on three different studies of the accretion of Solar material onto WDs and show that the results of $(36$; 37) must be revised.

\section{The Stellar Evolution Codes: NOVA and MESA}

The codes used are NOVA ( $45 ; 46 ; 47 ; 48 ; 49 ; 50$, and references therein) and MESA (51; 52; 53 , and references therein). NOVA is a one-dimensional (1-D), implicit, hydrodynamic, computer code which has been well tested against a number of standard problems and weapon codes (45; 46). It includes a nuclear reaction network that has been extended to 187 nuclei (up to ${ }^{64} \mathrm{Ge}$ and including the pep reaction), the OPAL opacities (54, and references therein), the Starlib nuclear reaction rates (55), the Timmes equations of state $(56 ; 57)$, and the nuclear reaction network solver developed by (58). NOVA also includes the (59) algorithm for mixing-length convection and the Potekhin electron conductivities described in (60, and references therein). These improvements have had the effect of changing the initial structures of the WDs so that, for a given mass, they have smaller radii and larger surface gravities.

One feature of NOVA is that it was designed to follow only the first evolution to the TNR. If we need to follow multiple events on an accreting WD, then we must switch to a different code. Our choice is MESA which solves the 1D fully coupled structure and composition equations governing stellar evolution. It is based on an implicit finite difference scheme with adaptive mesh refinement 
and sophisticated time step controls; state-of-the-art modules provide equations of state, opacity, nuclear reaction rates, element diffusion, boundary conditions, and changes to the mass of the star $(51 ; 52 ; 53)$. The equation of state is the 2005 update of the OPAL EOS (61) with an extension to lower temperatures and densities of the SCVH EOS (62). This EOS is supplemented with the HELM EOS (57) and the PC EOS (63) for the regimes where they are valid. The choice of opacity is the OPAL opacities (54) with the low temperature opacities of (64) and the electron conduction opacities of (60).

\section{Simulations of Thermonuclear Runaways that result in the Growth of the White Dwarf's Mass}

\subsection{Simulations with NOVA}

In this subsection we report on the first of two studies that investigated the consequence of a WD accreting stellar material with a Solar composition from a secondary donor star. We follow that with a study of accretion onto a CO WD but assume that mixing occurs during the TNR as has been found in the multi-D studies. Many types of close binary systems with a WD primary are suggested to be the progenitors for SN Ia explosions; thus we have modeled accretion onto a wide range of WD masses with a wide range of accretion rates. NOVA was used to study the consequences of accretion onto WDs with masses of $0.4 \mathrm{M}_{\odot}, 0.7 \mathrm{M}_{\odot}, 1.0 \mathrm{M}_{\odot}, 1.25 \mathrm{M}_{\odot}$, and $1.35 \mathrm{M}_{\odot}$, two initial WD luminosities $\left(4 \times 10^{-3} \mathrm{~L}_{\odot}\right.$ and $\left.10^{-2} \mathrm{~L}_{\odot}\right)$, and seven mass accretion rates ranging from $2 \times 10^{-11} \mathrm{M}_{\odot}$ $\mathrm{yr}^{-1}$ to $2 \times 10^{-6} \mathrm{M}_{\odot} \mathrm{yr}^{-1}$.

All simulations resulted in a TNR which either ejected some material or, after some time, the WD radius grew to $\sim 10^{12} \mathrm{~cm}$ and we halted the evolution. These calculations showed that the sequences exhibited the (65) thin shell instability. In general, the low mass WDs did not eject any mass while the high mass WDs ejected a small fraction of their accreted material (a maximum of $\sim 4 \%$ for the $1.25 \mathrm{M}_{\odot}$ sequences but ranging down to $\sim 0.1 \%$ for the $0.7 \mathrm{M}_{\odot}$ sequences). Therefore, the WDs are growing in mass as a result of the accretion of Solar composition material and no enrichment from core material.

Our studies (and those of others) show that most of the time to the TNR is spent in, and most of the mass is accreted during, the phase when the principle nuclear burning process is the protonproton chain. During this phase, there is a competition between the radiative diffusion time to the surface and electron degenerate conductivity that is transporting some energy into the interior. One reaction that affects the energy production during the proton-proton chain is the pep reaction $\left(p+e^{-}+p \rightarrow d+v: 66 ; 67\right)$. While for solar models the energy generation from the pep reaction is unimportant save for neutrino losses, in the WD envelope the density can reach, or exceed, values of $10^{4} \mathrm{~g} \mathrm{~cm}^{-3}$. At these densities, the rate of energy generation is larger than in those simulations done without the inclusion of the pep reaction. Therefore, calculations which include the pep reaction accrete less material then calculations where it is neglected (all other parameters held constant: 49). At this time in the evolution the energy production depends both on the temperature $\left(\sim \mathrm{T}^{4-6} \mathrm{~K}\right)$ and the hydrogen mass fraction (the energy generation: $\varepsilon \sim X^{2}$ ) and this is the phase when most of the mass is accreted. The evolution to the TNR begins to accelerate when the peak temperature in the nuclear burning region reaches $\sim 1.5 \times 10^{7} \mathrm{~K}$ but convection does not start until the temperature in 
the nuclear burning region has reached $\sim 3 \times 10^{7} \mathrm{~K}$ when the carbon-nitrogen-oxygen cycle (CNO) is producing all the nuclear energy.

Figure 1 shows the log of the difference between the mass accreted and the mass lost. Each point is the amount of accreted (less ejected) mass divided by the time to reach the TNR for the given simulation. The lines connect the points for the same $\dot{M}$. The log of the $\dot{M}$ used in these simulations is given along the column on the left of the figure.

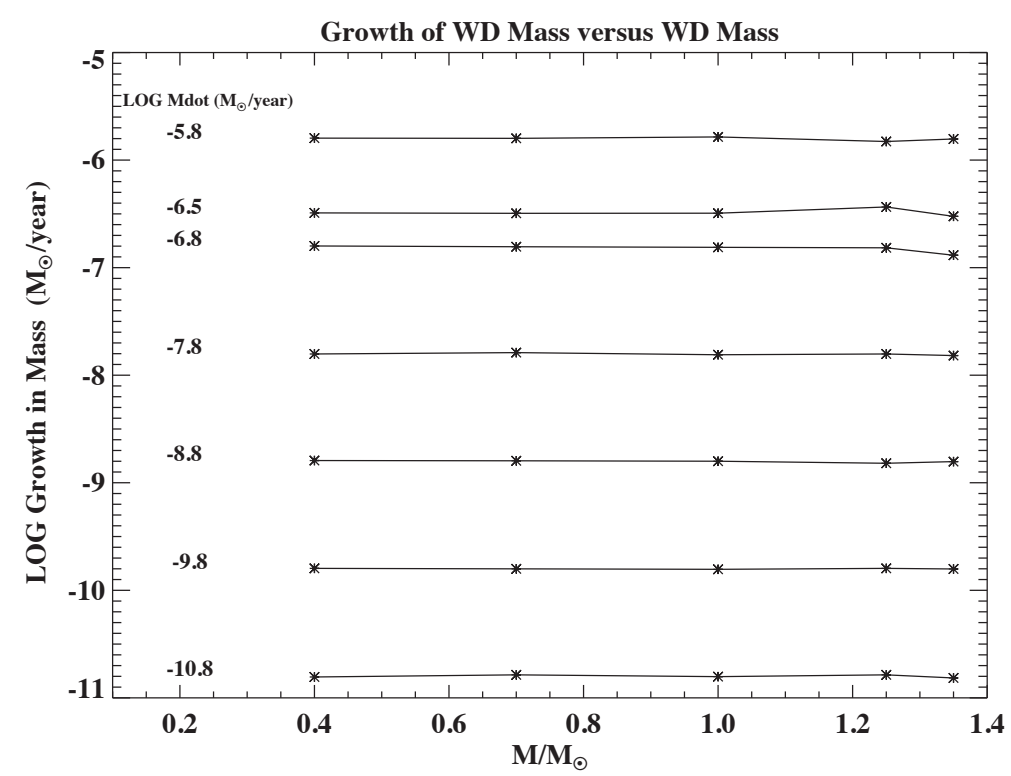

Figure 1: The log of the difference between the mass accreted and the mass lost. The log of the growth in mass (in units of $\mathrm{M}_{\odot} \mathrm{yr}^{-1}$ ) is displayed as a function of WD mass for each of the simulations. Each point is the amount of accreted (less ejected) mass divided by the time to reach the TNR for the given simulation. The lines connect the points for the same $\dot{M}$ and the $\log$ of $\dot{M}$ is given along a column on the left of the figure.

Figure 2 shows the accretion time to the TNR for some of our simulations. Clearly, as the WD mass increases, the accretion time decreases for the same $\dot{M}$. This is because higher mass WDs initiate the TNR with a smaller amount of accreted mass. Given the existence of Recurrent Novae (RNe) and Symbiotic Novae with recurrence times ranging from a few years (U Sco) to about 20 years (RS Oph) or longer (T Pyx, V407 Cyg, and T CrB), Figure 2 shows that it is possible for RNe to occur on WDs with masses as low as $0.7 \mathrm{M}_{\odot}$. Although it is often claimed that only the most massive WDs have recurrence times short enough to agree with the observations of $\mathrm{RNe}$, this is not the case and basing WD mass determinations for RNe purely on short recurrence times is incorrect. Note that it is also possible for a RN outburst to occur on a high mass WD for an extremely broad range of $\dot{M}$. Finally, for the most massive WDs, the recurrence period can be less than a year which suggests that the RN in M31 with an outburst period less than or about a year $(68 ; 69)$ must be occurring on a WD that is close to the Chandrasekhar Limit. 


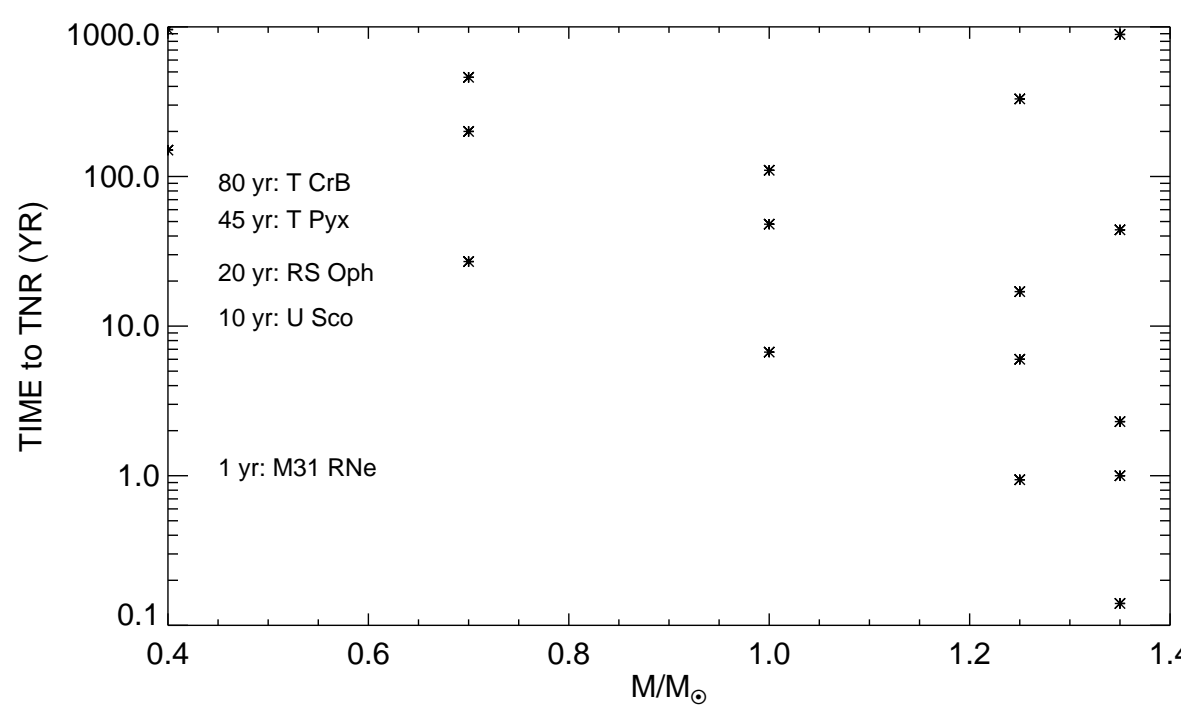

Figure 2: The accretion time to the TNR as a function of WD mass. Each of the data points is for a different $\dot{M}$ and the value of $\dot{M}$ increases downward for each WD mass. The time to the TNR decreases downward and to the right since it takes less mass to initiate the TNR as the WD mass increases. This plot also shows the approximate recurrence times for some of the best known RNe. The location of each $\mathrm{RN}$ indicates its approximate recurrence time. We note the position of the M31 RN which explodes on yearly time scale. This plot shows that not only is it possible for $\mathrm{RNe}$ outbursts to occur on low mass WDs but they can also occur for a broad range of $\dot{M}$ on higher mass WDs.

The evolution is halted when the outer radius reaches $\sim 10^{12} \mathrm{~cm}$ and the expanding material has become optically thin. The lower mass WD takes years to evolve to $\sim 10^{12} \mathrm{~cm}$ while the higher mass WDs take only days. The most extreme result is for a $0.4 \mathrm{M}_{\odot}$ WD for which it takes more than one year for the expanding material to become luminous and hotter. There is an initial long decline and then a slow recovery which is caused by the conversion of some of the internal energy, produced by ongoing nuclear burning near the surface, being transformed into the potential energy necessary for the material to climb out of the potential well of the WD.

While only a small amount of mass is lost in some of the simulations, that amount would increase if the effects of common envelope evolution in the binary system were included in the simulations. Finally, as the mass of the WD increases, the accreted mass necessary to reach the TNR declines from $6 \times 10^{-4} \mathrm{M}_{\odot}\left(0.4 \mathrm{M}_{\odot}\right)$ to $6 \times 10^{-6} \mathrm{M}_{\odot}\left(1.35 \mathrm{M}_{\odot}\right)$.

While the simulations with NOVA were done with fully 1-dimensional hydrodynamics, they were only able to follow the first outburst on the WD. In all cases a TNR occurred and caused the outer layers to begin expanding to large radii. In a few cases the outermost mass zones reached escape velocity and became optically thin. The amount of material lost, if any, was tabulated but the escaping zones were not removed from the mass zoning because, even if they were escaping, they still exerted a numerical pressure on the underlying layers and if removed would cause excessive mass loss. 


\subsection{Simulations with MESA}

In this subsection we report on simulations done with MESA, because it is capable of following multiple outbursts on an accreting WD and, thereby, determining the secular evolution of the WD. The MESA studies followed the evolution of the WD as it accreted stellar material with a Solar composition and experienced a large number of TNRs resulting in ejection, mass loss, return to quiescence, and then the renewal of accretion. The treatment of mass loss after the TNR is important in following the long term behavior of the WDs, determining if mass growth occurs, and, if so, how rapidly. In order to determine the effects of different mass loss prescriptions, the evolution of a $1.35 \mathrm{M}_{\odot}$ WD accreting at a rate of $1.6 \times 10^{-9} \mathrm{M}_{\odot} \mathrm{yr}^{-1}$ was followed using three different mass loss prescriptions. These were (1) Eddington wind mass loss (70); (2) determining the amount of mass ejected when the outer layers exceeded the escape velocity, had become optically thin, and the surface radius equaled $10^{12} \mathrm{~cm}$ (this is the prescription in NOVA); and (3) Roche Lobe overflow with an assumed ejection rate of $10^{-6} \mathrm{M}_{\odot} \mathrm{yr}^{-1}$ at a radius of $1.0 \mathrm{R}_{\odot}$.

The major differences in the results using these 3 mechanisms was that the accretion efficiency (defined as the mass accreted minus the mass ejected divided by the mass accreted over a flash cycle) was $\sim 13 \%$ for the Eddington wind prescription, $\sim 15 \%$ to $20 \%$ for Roche lobe overflow, and $\sim 90 \%$ for the method used in NOVA. Therefore, in comparison with the NOVA studies, the Eddington wind method ejects a larger amount of the accreted mass than NOVA so that the WD grows in mass more slowly. However, the Eddington wind method was used in the MESA simulations because it is more easily implemented for following repeated TNRs.

Initial WD masses of $0.7 \mathrm{M}_{\odot}, 1.0 \mathrm{M}_{\odot}$ and $1.35 \mathrm{M}_{\odot}$ were used. All WDs consisted of bare $\mathrm{CO}$ cores $(\mathrm{C}=0.357, \mathrm{O}=0.619)$ at the beginning of accretion. The mass accretion rates were chosen to be $1.6 \times 10^{-10} \mathrm{M}_{\odot} \mathrm{yr}^{-1}, 1.6 \times 10^{-9} \mathrm{M}_{\odot} \mathrm{yr}^{-1}, 1.6 \times 10^{-8} \mathrm{M}_{\odot} \mathrm{yr}^{-1}$, and $1.6 \times 10^{-7} \mathrm{M}_{\odot} \mathrm{yr}^{-1}$ Other accretion rates were used in order to separate different regimes of behavior when needed. Again, the composition of the accreted material was Solar. All simulations were run for either many TNR cycles or the time required for long-term behavior to become evident.

Figure 3 shows the WD mass as a function of time for 4 different mass accretion rates for a $1.35 \mathrm{M}_{\odot}$ WD accreting at $1.6 \times 10^{-9} \mathrm{M}_{\odot} \mathrm{yr}^{-1}, 1.6 \times 10^{-8} \mathrm{M}_{\odot} \mathrm{yr}^{-1}, 1.6 \times 10^{-7} \mathrm{M}_{\odot} \mathrm{yr}^{-1}$, and $6.4 \times$ $10^{-7} \mathrm{M}_{\odot} \mathrm{yr}^{-1}$. For the three lowest mass accretion rates, after the initial growth to the first TNR, the evolutionary sequence settles into a recurring pattern of mass accretion resulting in TNRs which eject a fraction of the accreted mass via an Eddington wind (70). Once the mass is lost, accretion resumes until the next TNR occurs. The evolution at $1.6 \times 10^{-7} \mathrm{M}_{\odot} \mathrm{yr}^{-1}$, exhibits a positive slope with the WD growing in mass at a rate of $\sim 3.0 \times 10^{-8} \mathrm{M}_{\odot} \mathrm{yr}^{-1}$. This implies an efficiency per cycle of approximately $20 \%$. The recurrence time for this sequence is almost short enough to agree with that of the M31 RN that is outbursting about once per year (69).

Using MESA, we found that in all cases the simulation evolves to a TNR but only a small amount of accreted material is ejected and the WDs are growing in mass. These studies show that the evolutionary sequences exhibit the (65) thin shell instability, which implies that steady burning does not occur. An expanded study of the stability of thin shells can be found in (71), who investigated the accretion of hydrogen-rich material onto WDs. Using their results, we find that our sequences begin in their stable region, but with continued accretion, evolve into instability. We identify these systems with those CVs (dwarf, recurrent, symbiotic novae) that show no core 

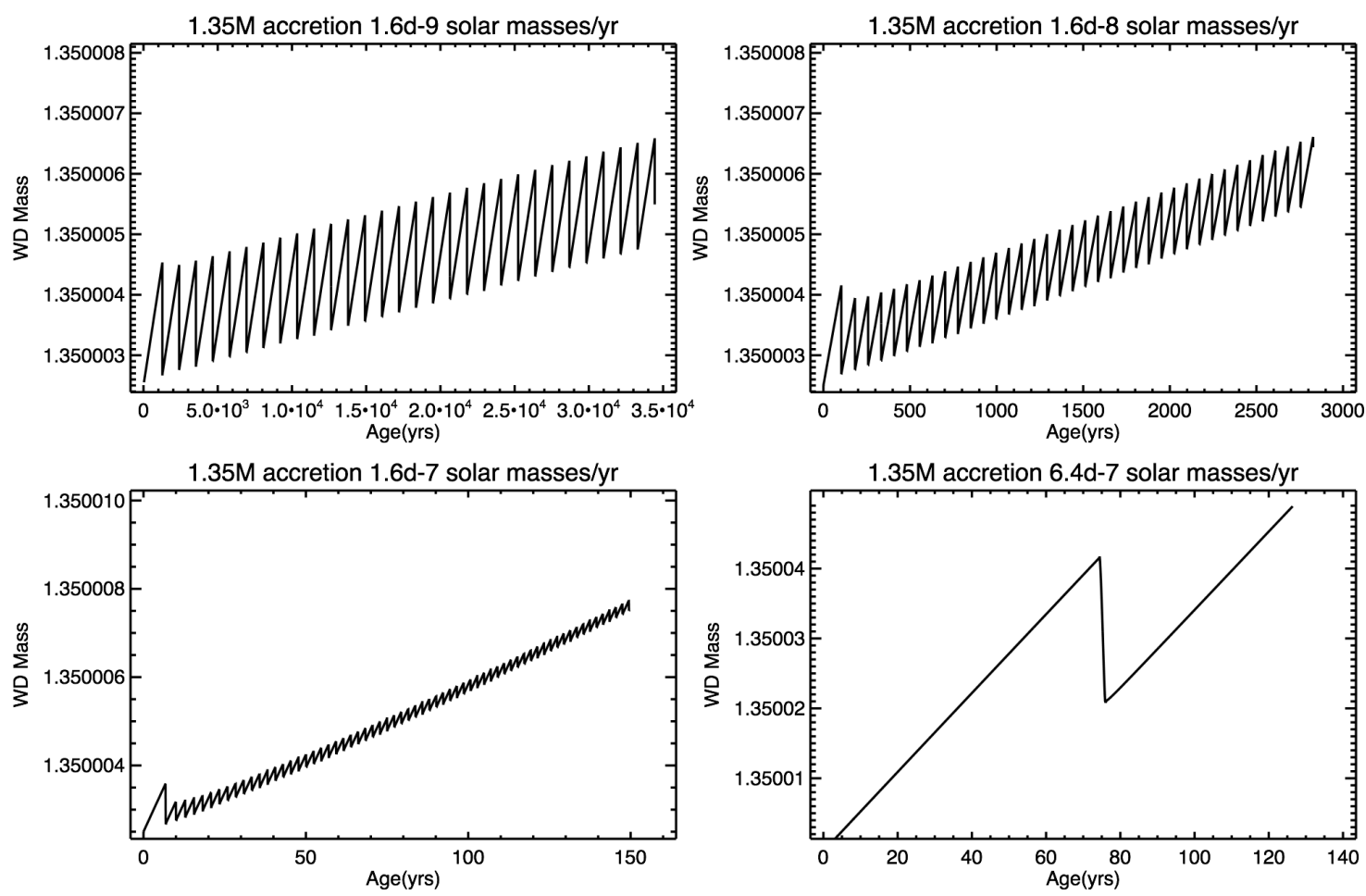

Figure 3: The WD mass as a function of time for 4 different mass accretion rates for a $1.35 \mathrm{M}_{\odot} \mathrm{WD}$ accreting at $1.6 \times 10^{-9} \mathrm{M}_{\odot} \mathrm{yr}^{-1}, 1.6 \times 10^{-8} \mathrm{M}_{\odot} \mathrm{yr}^{-1}, 1.6 \times 10^{-7} \mathrm{M}_{\odot} \mathrm{yr}^{-1}$, and $6.4 \times 10^{-7} \mathrm{M}_{\odot} \mathrm{yr}^{-1}$. For the three lowest mass accretion rates, after the initial growth to the first TNR, the evolutionary sequence settles into a recurring pattern of mass accretion resulting in TNRs which eject a fraction of the accreted mass via an Eddington wind (70). Once the mass is lost, accretion resumes until the next TNR occurs. Concentrating on the evolution at $1.6 \times 10^{-7} \mathrm{M}_{\odot} \mathrm{yr}^{-1}$, we see that the positive slope shows that the WD is growing in mass at a rate of $\sim 3.0 \times 10^{-8} \mathrm{M}_{\odot} \mathrm{yr}^{-1}$. This implies an efficiency per cycle of approximately $20 \%$. The recurrence time for this sequence is almost short enough to agree with that of the M31 RN that is outbursting about once per year (69).

material either on the surface of the WD or in their ejecta. Our results could explain the findings of (72), who report that the WDs in CVs are growing in mass. In addition, the best studied dwarf novae have WD masses larger than the canonical value of $\sim 0.6 \mathrm{M}_{\odot}$. These are $\mathrm{U}$ Gem $\left(1.2 \mathrm{M}_{\odot}: 73\right)$, SS Cyg $\left(0.8 \mathrm{M}_{\odot}: 74\right)$, IP Peg $\left(1.16 \mathrm{M}_{\odot}: 75\right)$, and Z Cam $\left(0.99 \mathrm{M}_{\odot}: 76\right)$. Therefore, it seems possible that some Dwarf Novae could be SN Ia progenitors if there is some means to prevent convection from mixing accreted with WD core material. For example, a thick helium layer from previous outbursts could act as a barrier to this mixing. Further work is warranted.

While the evolution shown in Figure 3 implies that a $1.35 \mathrm{M}_{\odot}$ WD will grow in mass with repeated hydrogen flashes, we also tested the evolution to determine if accretion at a higher rate would cause the WD to grow in radius to that of a red giant as proposed by $(37 ; 36)$. Therefore, we evolved a sequence with $\dot{\mathrm{M}}=6.4 \times 10^{-7} \mathrm{M}_{\odot} \mathrm{yr}^{-1}$. That result is shown in the lower right hand panel of Figure 3. After approximately seventy-five years of accretion, the WD undergoes a helium flash which does not eject any material. The radius of the WD expands to $0.056 \mathrm{R}_{\odot}$ before contracting 
and the peak surface velocity reaches only $10 \mathrm{~km} \mathrm{~s}^{-1}$. This radius is less than the typical Roche Lobe radius of a WD in a CV system. Therefore, such helium flashes do not stop the growth in the mass of the WD from either Roche Lobe overflow or envelope ejection. After the initial helium flash, the WD undergoes succeeding helium flashes roughly every 75 years. During these flashes, the WD grows in mass at a rate of $\sim 2.6 \times 10^{-7} \mathrm{M}_{\odot} \mathrm{yr}^{-1}$. The helium flashes are not violent because mass is being lost via an Eddington wind. Thus, a helium flash is not the dynamic mass ejection event that occurs with the mass loss prescription used in NOVA.

Our conclusion from this part of the study, where we accrete Solar composition material onto WDs of various masses and use various accretion rates (with both NOVA and MESA), is that the CO WD in a typical CV system is growing in mass toward the Chandrasekhar Limit.

\subsection{Accretion onto CO WDs where Mixing of Core with Accreted material is assumed}

We continue with new simulations of accretion onto CO WDs where we assume that mixing of accreted material occurs with core material. We use NOVA for this new study and note that in an earlier study of accretion onto CO white dwarfs (77) we were primarily concerned with those $\mathrm{CNe}$ that formed dust. Since that time an additional number of $\mathrm{CNe}$ have been observed that formed dust and, in addition, a number of pre-solar grains have been found that may have been produced in $\mathrm{CNe}$ explosions. There are now a number of studies of grain formation in $\mathrm{CN}$ outbursts $(78 ; 79 ; 80 ; 81 ; 82)$. In addition, we now use the STARLIB reaction rate library $(55)$ which improves the rates for many of the light nuclear reactions.

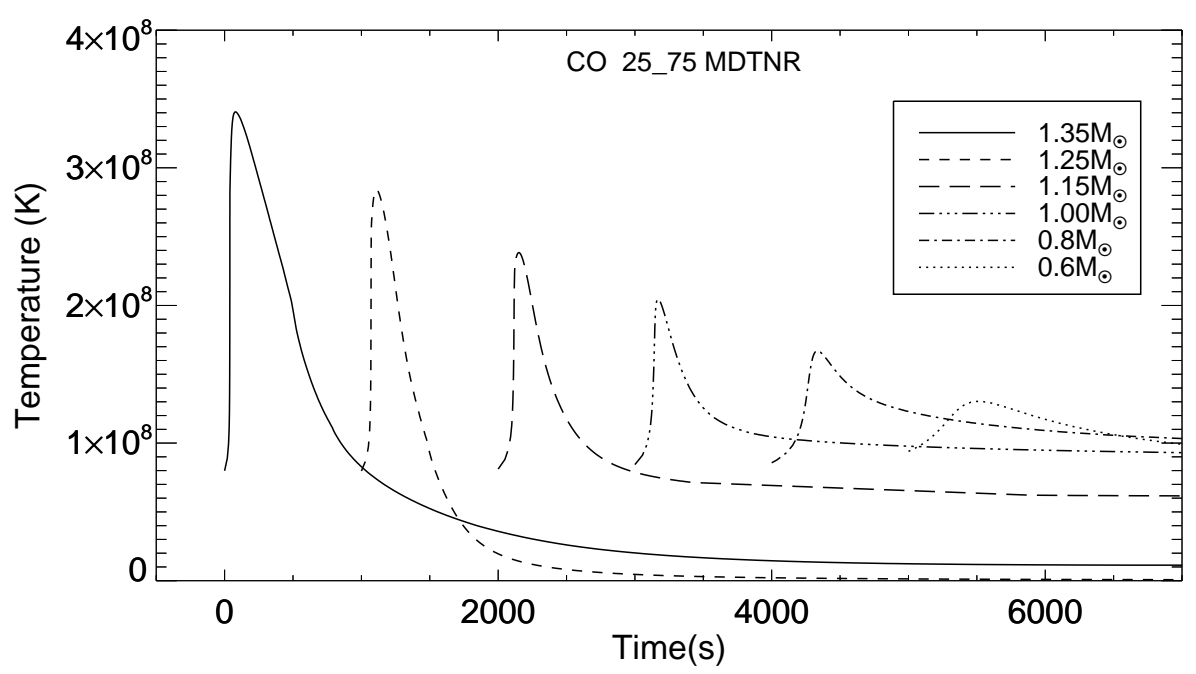

Figure 4: The variation with time of the temperature in the deepest hydrogen-rich zone around the time when peak temperature occurs for the simulations with $25 \%$ core material and $75 \%$ accreted material. We have plotted the results for all six simulations which only differ by WD mass. The identification with mass is given in the box. The curve for each sequence has been shifted slightly in time to improve its visibility. As expected, the peak temperature achieved in each simulation is an increasing function of WD mass.

For this study we accreted at a rate of $1.6 \times 10^{-10} \mathrm{M}_{\odot} \mathrm{yr}^{-1}$ onto CO WDs (core composition was $50 \%{ }^{12} \mathrm{C}$ and $50 \%{ }^{16} \mathrm{O}$ ) with masses of $0.6 \mathrm{M}_{\odot}, 0.8 \mathrm{M}_{\odot}, 1.00 \mathrm{M}_{\odot}, 1.15 \mathrm{M}_{\odot}, 1.25 \mathrm{M}_{\odot}$, and 


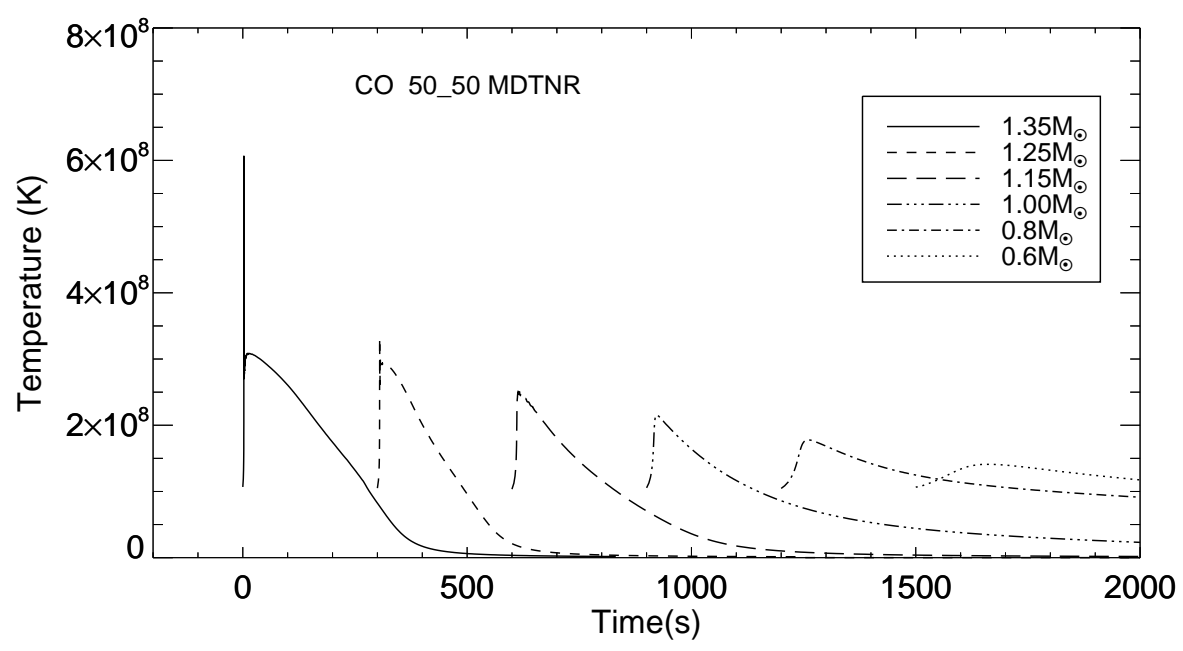

Figure 5: The variation with time of the temperature for the simulations with $50 \%$ core matter and $50 \%$ accreted matter for the six WD masses that we studied. Because there is significantly more ${ }^{12} \mathrm{C}$ in these simulations when we switch to this composition, the peak temperature is higher for each of these masses than in the $25 \%$ core matter simulations. In fact, the $1.35 \mathrm{M}_{\odot}$ simulation reaches a peak temperature exceeding $6 \times 10^{8} \mathrm{~K}$ and the temperature rise to the peak of the TNR is so rapid that a shock forms and moves through the envelope in a few seconds. Note that the evolution time for this series of sequences is shorter than that in Figure 4.

$1.35 \mathrm{M}_{\odot}$. Although it is commonly assumed that a CO WD should not have a mass exceeding $\sim$ $1.15 \mathrm{M}_{\odot}$, our simulations, which suggest that WDs can accrete mass and grow to the Chandrasekhar Limit, indicate that there should be massive CO WDs in CN systems. In support of this assumption, we note that LMC 1991 exhibited a super Eddington luminosity for more than 2 weeks (83) which probably requires a WD mass exceeding $\sim 1.35 \mathrm{M}_{\odot}$. Moreover, the WDs in 4 of the nearest CVs are more massive than the canonical value for single WDs of $0.6 \mathrm{M}_{\odot}$.

There is a major change in the way we treat the composition of the accreting material in this study as compared to our previous studies. In our previous work, except for (79), we assume that the mixing of core and accreted material occurs from the beginning of the simulation so that we accrete half core and half Solar (accreted) material (49, and references therein). From a variety of tests that will be published elsewhere, we came to realize that the initial ${ }^{12} \mathrm{C}$ abundance strongly influences the amount of accreted material since the more ${ }^{12} \mathrm{C}$ present in the material the more rapidly the simulation evolves to a TNR with less accreted mass.

In order to increase the amount of accreted material, we assume the results of the multidimensional studies that show that sufficient material from the core is dredged-up into the accreted envelope by convectively associated instabilities after the TNR is well underway (84, and references therein). Therefore, we first accreted a Solar Mixture until the temperature in the nuclear burning regime exceeded $\sim 3.0 \times 10^{7} \mathrm{~K}$ and convection was well underway. The accretion of Solar material onto WDs of various masses and mass accretion rates has been published elsewhere $(40 ; 85 ; 86)$. At this point in the evolution we switched to the mixed composition (this includes both nuclei abundances and the associated microphysics) that we have used in our previous studies 


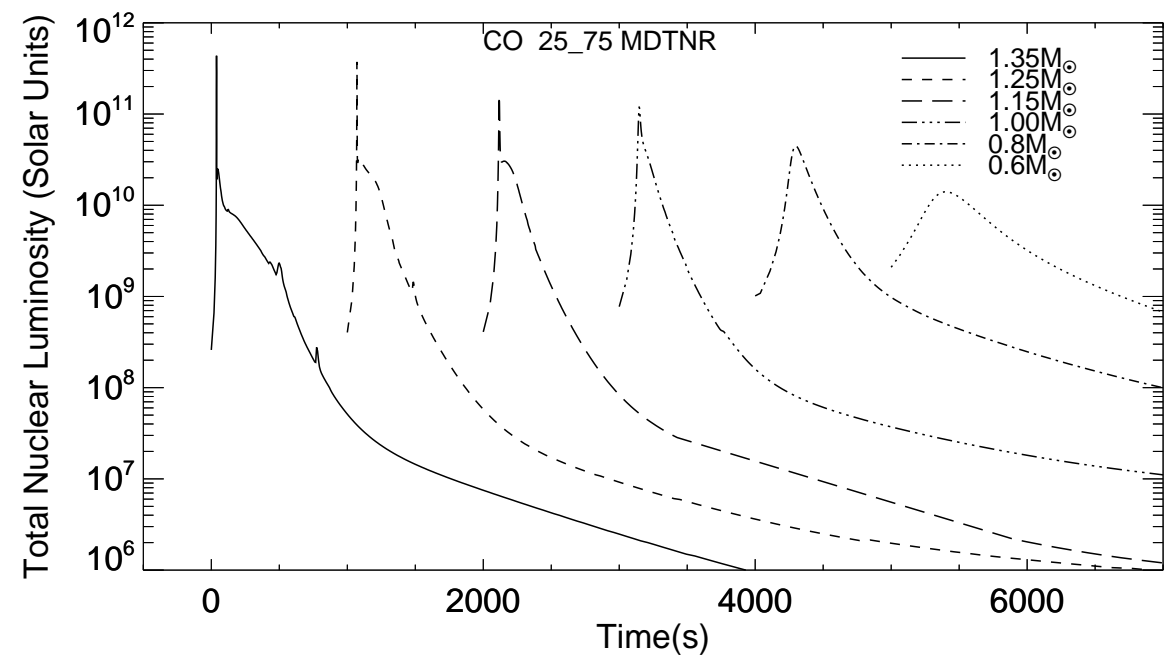

Figure 6: The variation with time of the total nuclear luminosity $\left(\mathrm{erg} \mathrm{s}^{-1}\right)$ in solar units $\left(\mathrm{L}_{\odot}\right)$ around the time of peak temperature during the TNR on both sets of simulations. We integrated over all zones taking part in the explosion to obtain the plotted numbers. The identification with each WD mass is given on the plot and the evolution time is the same as for each of the temperature plots. The small "glitches" that appear on the decline are caused by convection moving in and out and bringing in small amounts of fresh nuclei to the nuclear burning regime.

(49; 50, and references therein) and proceed through the rest of the evolution to peak temperature and the decline. In addition, we assume the results of (87) and use an accreted plus core composition of either $25 \%$ core and $75 \%$ accreted material or $50 \%$ core and $50 \%$ accreted material. Those are identified on the plots as either $25 \_75$ or 50_50. The same initial model is used for the two compositions: thermal structure, spatial structure, and total amount of accreted mass.

The following plots show the evolution of the various simulations done for this paper. The acronym: MDTNR stands for Mixing During the TNR. Other studies to be reported elsewhere describe either the Solar accretion results or the Mixing From Beginning (MFB) results. Figure 4 shows the variation with time of the temperature in the deepest hydrogen-rich zone around the time of peak temperature in the simulation. This plot shows the evolution for the simulations with $25 \%$ core matter and $75 \%$ accreted matter. As expected, the simulation on a WD with a mass of $1.35 \mathrm{M}_{\odot}$ reaches the highest temperature of $3.4 \times 10^{8} \mathrm{~K}$ while the simulation on the lowest mass WD that we studied, $0.6 \mathrm{M}_{\odot}$, reaches the lowest peak temperature of $1.3 \times 10^{8} \mathrm{~K}$. Because the abundance of ${ }^{12} \mathrm{C}$ is less in these simulations than in the $50 \%$ core $-50 \%$ accreted matter simulations, the evolution takes longer to go through the peak of the TNR and begin the decline to quiescence so that the time axis is longer than in Figure 5. Figure 5 shows the same evolution but for the simulations with 50\% core and 50\% accreted material. The peak temperature is higher for each of these masses because there is significantly more ${ }^{12} \mathrm{C}$. The $1.35 \mathrm{M}_{\odot}$ calculation reaches a peak temperature exceeding $6 \times 10^{8} \mathrm{~K}$ and the temperature rise to the peak of the TNR is so rapid that a shock forms and moves through the envelope in a few seconds.

The next two figures show the variation with time of the total nuclear luminosity ( $\mathrm{erg} \mathrm{s}^{-1}$ ) in solar units $\left(\mathrm{L}_{\odot}\right)$ around the time of peak temperature during the TNR on both sets of simulations. 


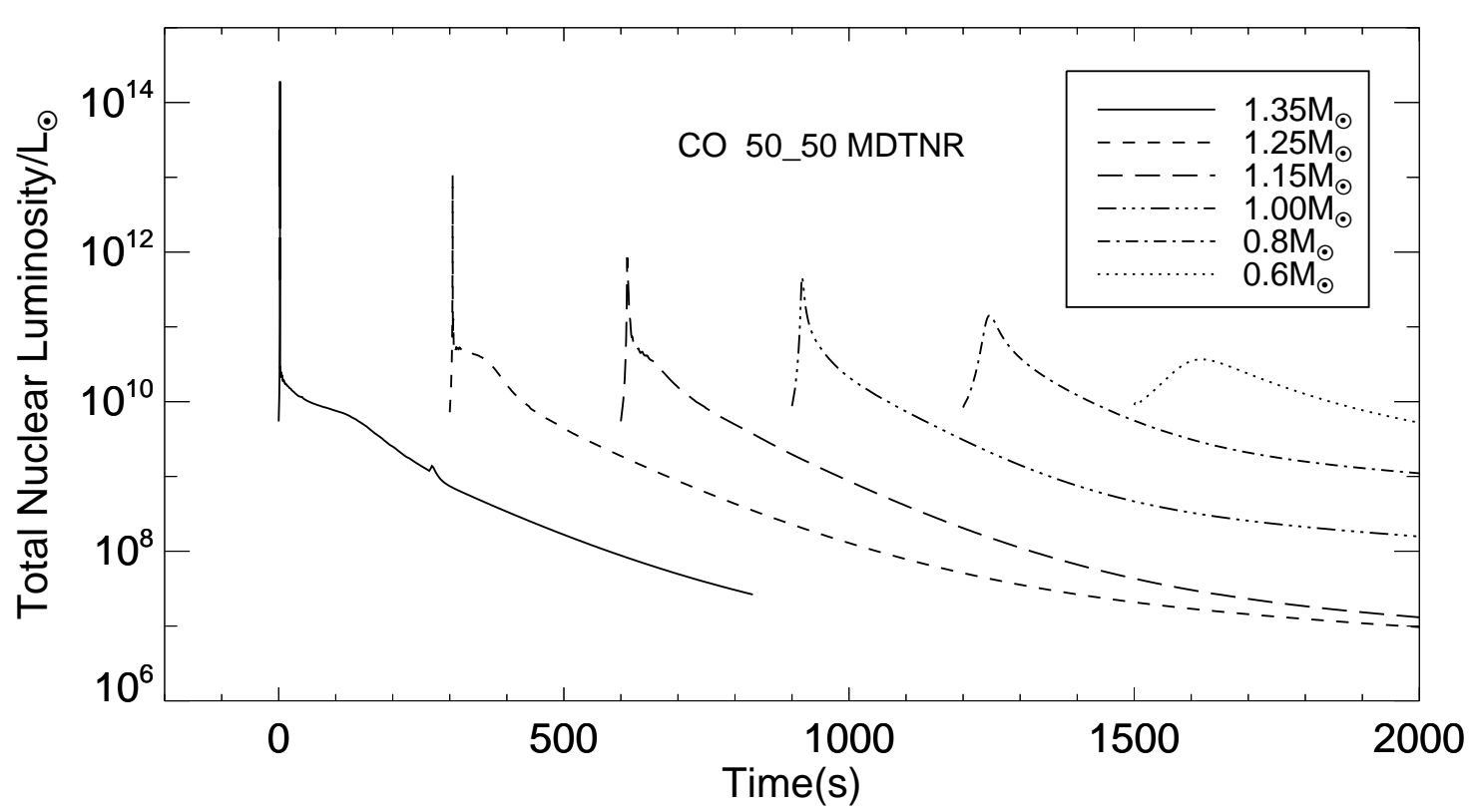

Figure 7: The same plot as in Figure 6 but for the simulation with 50\% core matter and 50\% accreted matter. Note that the horizontal and vertical axes differ in these two plots.

We integrated over all zones taking part in the explosion to obtain the plotted numbers. The identification with each WD mass is given on the plot and the evolution time is the same as for each of the temperature plots. The small "glitches" that appear on the decline are caused by convection moving in and out and bringing in small amounts of fresh nuclei to the nuclear burning regime. Figure 6 shows the evolution of nuclear energy production for the simulations with $25 \%$ core matter (and 75\% accreted matter) while Figure 7 shows the plot for 50\% core matter (and 50\% accreted matter). Note that both the horizontal axes and vertical axes are different for each plot but agree with those in Figure 4 and Figure 5, respectively.

There are two major results that we present in the next two figures. First, is the amount of ${ }^{7} \mathrm{Be}$ produced during the TNR. Since ${ }^{7} \mathrm{Be}$ decays to ${ }^{7} \mathrm{Li}$ with a $\sim 53$ day half-life, it is also a measure of the amount of lithium produced in the outburst. Clearly, $\mathrm{CN}$ should produce a great deal of ${ }^{7} \mathrm{Li}$ and may be the galactic source of this nucleus as originally predicted by (88) for red giants and (89) for $\mathrm{CN}$ explosions, with a more recent study by (90). The discovery of both ${ }^{7} \mathrm{Be}$ and ${ }^{7} \mathrm{Li}$ in recent high dispersion spectroscopy of bright $\mathrm{CN}$ near the peak of their outburst (reviewed in the next section which also includes a new discovery of ${ }^{7} \mathrm{Li}$ ) has required us to redo the earlier calculations with updated compositions and microphysics. Figure 8 shows our predicted ${ }^{7} \mathrm{Be}$ abundances in the ejecta as a function of WD mass for all the $\mathrm{CO}$ enriched sequences done for this paper. We plot the ratio of ${ }^{7} \mathrm{Be}+{ }^{7} \mathrm{Li}$ to ${ }^{7} \mathrm{Li} \odot$. The TNR reaches sufficiently high temperatures to deplete the original ${ }^{7} \mathrm{Li}$ present in the accreted material so that the ratio is really that of the ejected ${ }^{7} \mathrm{Be}$. For example, the ejected ${ }^{7} \mathrm{Li}$ abundances by mass fraction are $\sim 10^{-13}$ while the ejected abundances of ${ }^{7} \mathrm{Be}$ are on the order of $10^{-5}$ (mass fraction). All our sequences eject enriched ${ }^{7} \mathrm{Be}$ and the amount of enrichment increases as a function of WD mass. We halt the evolution before the ${ }^{7} \mathrm{Be}$ can decay to ${ }^{7} \mathrm{Li}$ so we only report the ${ }^{7} \mathrm{Be}$ abundance. 


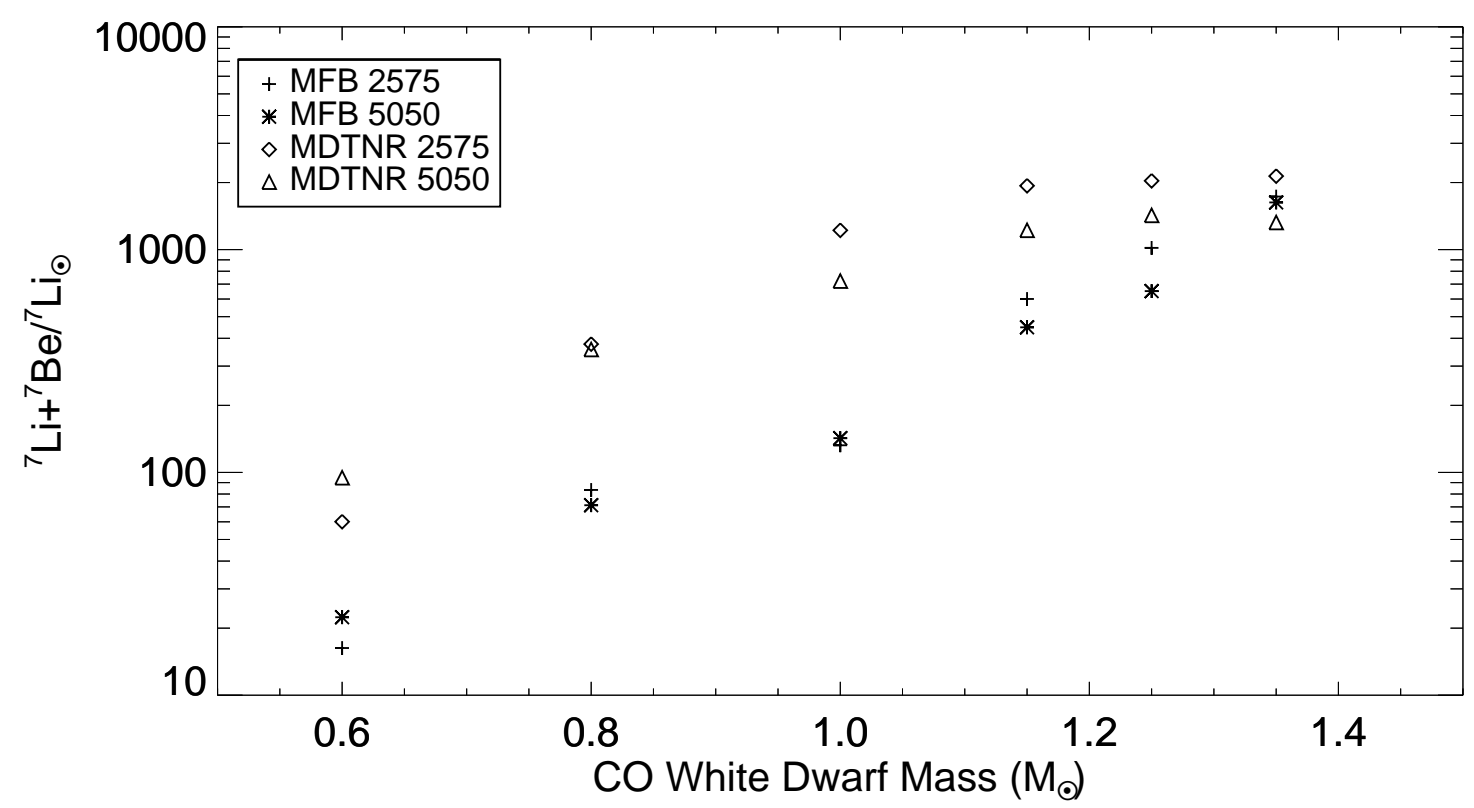

Figure 8: The predicted ${ }^{7} \mathrm{Be}$ abundances in the ejecta as a function of WD mass for all the $\mathrm{CO}$ enriched sequences done for this paper. While we plot the ratio of ${ }^{7} \mathrm{Be}+{ }^{7} \mathrm{Li}$ to ${ }^{7} \mathrm{Li}_{\odot}$, the TNR reaches sufficiently high temperatures to deplete the initial ${ }^{7} \mathrm{Li}$ present in the accreted material so that the ratio is that of the ejected ${ }^{7} \mathrm{Be}$.(Mixing From Beginning = MFB)

Figure 9 shows the second major result: the percent of ejected mass (ejected over accreted) as a function of WD mass for all the sequences that we evolved. The bottom of the vertical axis looks strange (a negative 20\%) because we need to emphasize the fact that most of our sequences ejected almost no material as a result of the TNR and, therefore, the WD is growing in mass toward the Chandrasekhar Limit. The only sequences that eject a significant amount of material are those where we mix after the TNR is underway (MDTNR). Examining the sequences with 25\% core matter and $75 \%$ accreted (Solar) composition we find that only the $1.25 \mathrm{M}_{\odot}$ sequence ejects a sufficient amount of material and the WD is probably losing mass as a result of the TNR and resulting explosion. Clearly, those sequences with $50 \%$ core matter and $50 \%$ accreted matter could have either the WD losing mass (WD masses exceeding $\sim 1.0 \mathrm{M}_{\odot}$ ) or gaining mass (WD mass $\leq$ $\left.1.0 \mathrm{M}_{\odot}\right)$. Therefore, we predict that for most observed $\mathrm{CO}$ classical novae the WD is gaining mass as a result of the explosion.

Finally, in this section we show the production plot for the $1.35 \mathrm{M}_{\odot}$ sequence with $25 \%$ core matter and $75 \%$ accreted matter (Figure 10). This plot is based on similar plots in (91). The production plots for the other sequences will be published elsewhere. While normally a production plot only shows the stable nuclei in the ejected material, for our simulations the ejecta abundance of ${ }^{7} \mathrm{Be}$ is important. As long known, the $\mathrm{CN}$ outburst on a $\mathrm{CO}$ WD ejects mainly the odd light isotopes. It shows that ${ }^{7} \mathrm{Be},{ }^{13} \mathrm{C},{ }^{15} \mathrm{~N},{ }^{17} \mathrm{O},{ }^{31} \mathrm{P},{ }^{33} \mathrm{~S},{ }^{35} \mathrm{Cl}$, and ${ }^{40} \mathrm{Ca}$ are overproduced in $\mathrm{CO}$ nova ejecta. 


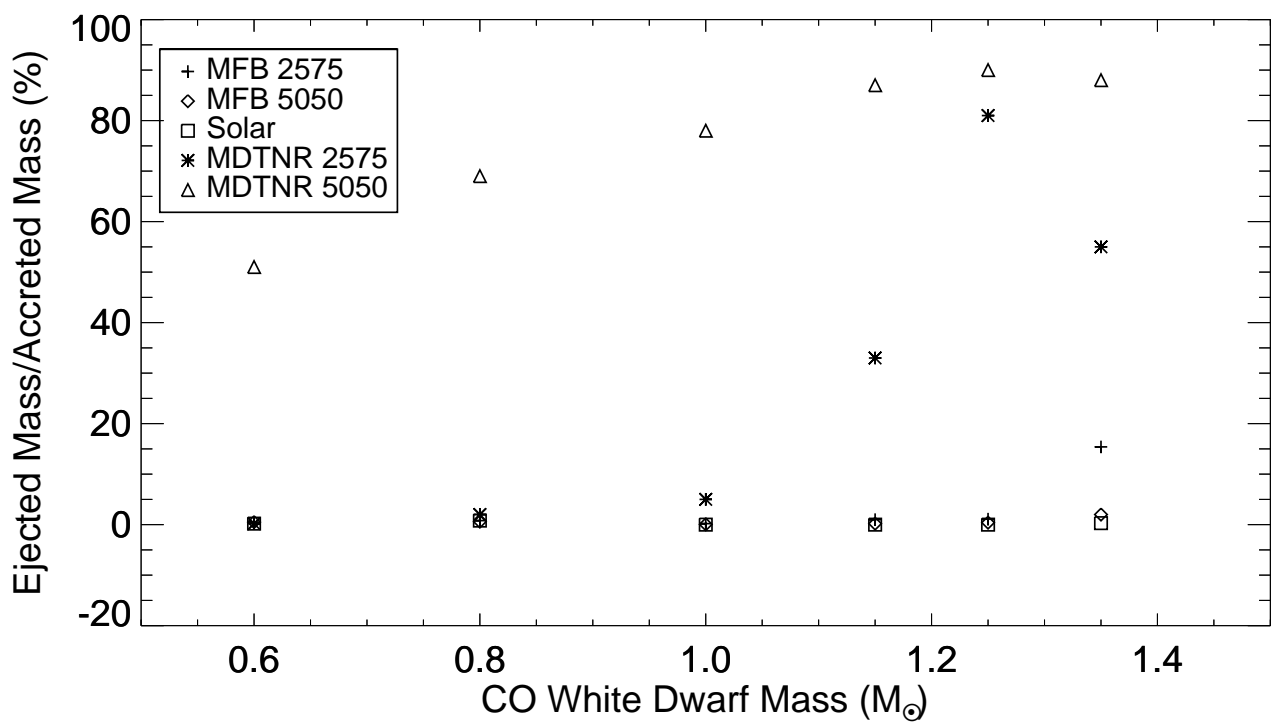

Figure 9: The percent of ejected mass divided by the accreted mass as a function of WD mass. The value of " -20 " on the bottom of the vertical axis was done to expand the vertical axis in order to show clearly that most of the sequences ejected almost no material as a result of the TNR and, therefore, the WD was growing in mass toward the Chandrasekhar Limit. The only sequences that eject a significant amount of material are those where we mix after the TNR is underway (MDTNR).

\section{5. ${ }^{7} \mathrm{Li}$ in $\mathrm{V5668} \mathrm{Sgr}$}

Given our predictions for enriched ${ }^{7} \mathrm{Be}$ which decays into ${ }^{7} \mathrm{Li}$, here we report on our (Wagner, Woodward, Starrfield et al. 2018 in preparation) second direct detection of spectral lines arising from explosive production of ${ }^{7} \mathrm{Li}$ I $6707.76 \AA$ in the post-outburst spectra of V5668 Sgr (Nova Sgr 2013 No. 2). The first detection of ${ }^{7} \mathrm{Li}$ was reported for V1369 Cen (92). V5668 Sgr was discovered by J. Seach (IAUC 9275) on 2015 Mar 15.634 UT ( $t_{0}=$ JD 2,457,097.134). A low resolution spectrum obtained by Powles (IAUC 9275) showed an $\mathrm{H} \beta$ emission line and an emerging Fe II emission line spectrum suggesting that it was an Fe II-type classical nova. The nova reached 5th magnitude in mid-March. After a slow decline for the first 80 days, the brightness declined $\sim 7$ mag as the result of dust formation (ATEL 7643, 7748, 7862, 7986) reaching a minimum on day 110. The light curve then recovered to 9 th mag by the end of our observations.

We observed V5668 Sgr on 30 nights between 2015 Apr 3 and 2016 June 5 UT. Our detections of Li were significant and persisted over the course of 7 nights (Figure 11 and Figure 12). The equivalent width of the Li line declined during the observations as the ionization of the ejecta increased. We did not detect this line by Day 42 of the outburst. Our discovery was based on high resolution optical spectroscopy using the Potsdam Echelle Polarimetric and Spectroscopic Instrument (PEPSI: 93) built for the $2 \times 8.4 \mathrm{~m}$ Large Binocular Telescope (LBT). PEPSI is the highest resolution spectrometer available on any 8-10 m class telescope. Our spectra covered all or part of the $3830 \AA$ to $9065 \AA$ region in three exposures at spectral resolutions of $43,000,120,000$ or 270,000 . Six cross dispersers cover six wavelength settings where two are observed simultaneously. 


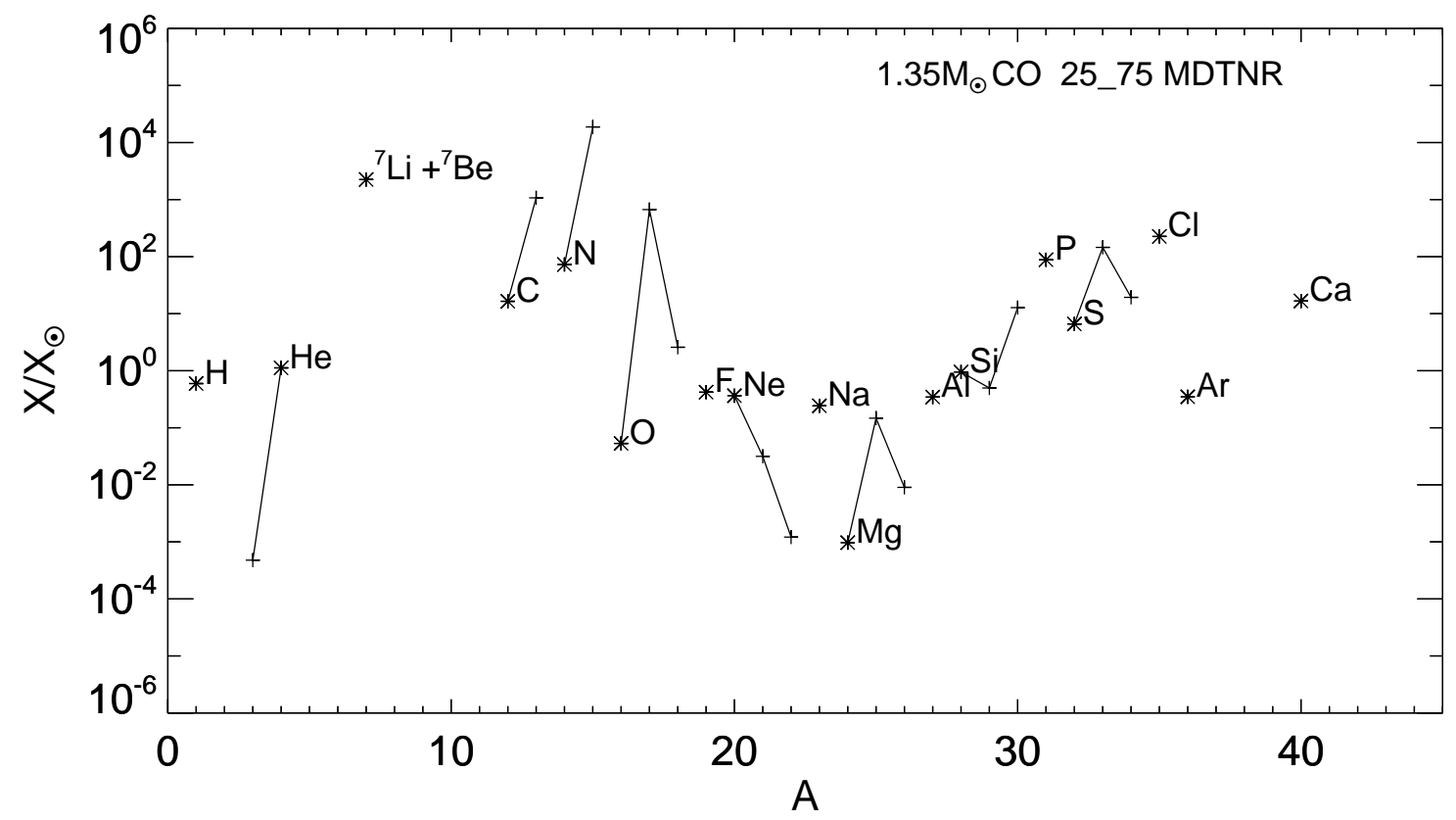

Figure 10: The abundances (mass fraction) of the stable isotopes from hydrogen to calcium in the ejecta for the $1.35 \mathrm{M}_{\odot}$ sequence. However, we include ${ }^{7} \mathrm{Be}$ in this plot because of its large overproduction. In fact, ${ }^{7} \mathrm{Li}$ is depleted during the evolution. The $x$-axis is the atomic mass and the $y$-axis is the logarithmic ratio of the abundance divided by the Solar abundance. As in (91), the most abundant isotope of a given element is designated by an " $*$ " and all isotopes of a given element are connected by solid lines. Any isotope above 1.0 is overproduced in the ejecta and a number of isotopes are significantly enriched in the ejecta.

PEPSI can also be fed by a 350m fiber running between the $1.8 \mathrm{~m}$ Vatican Advanced Technology Telescope (VATT) and the LBT. (94) observed ${ }^{7} \mathrm{Be}$ in V5668 Sgr on day 69 and a comparison of their and our results gives for the first time a measurement of the ${ }^{7} \mathrm{Be} /{ }^{7} \mathrm{Li}$ mass fraction ratio of 4200 on Day 13. This strongly suggests that ${ }^{7} \mathrm{Be}$ was created in the TNR as predicted by (89) and (90).

\section{Conclusions}

1. The amount of accreted material is an inverse function of the initial abundance of ${ }^{12} \mathrm{C}$.

2. Therefore, accreting Solar material (rather than mixed) allows for more matter to be accreted. In addition, reducing the metallicity to values in agreement with the LMC, SMC, or even lower also reduces the initial ${ }^{12} \mathrm{C}$ allowing more material to be accreted before the TNR is initiated $(95 ; 79)$. New studies with lower metallicities are in progress.

3. Therefore, either no mixing with the core or mixing too early with the $\mathrm{CO}$ core results in an outburst that is less violent and hardly any material (accreted plus core) is ejected during the 

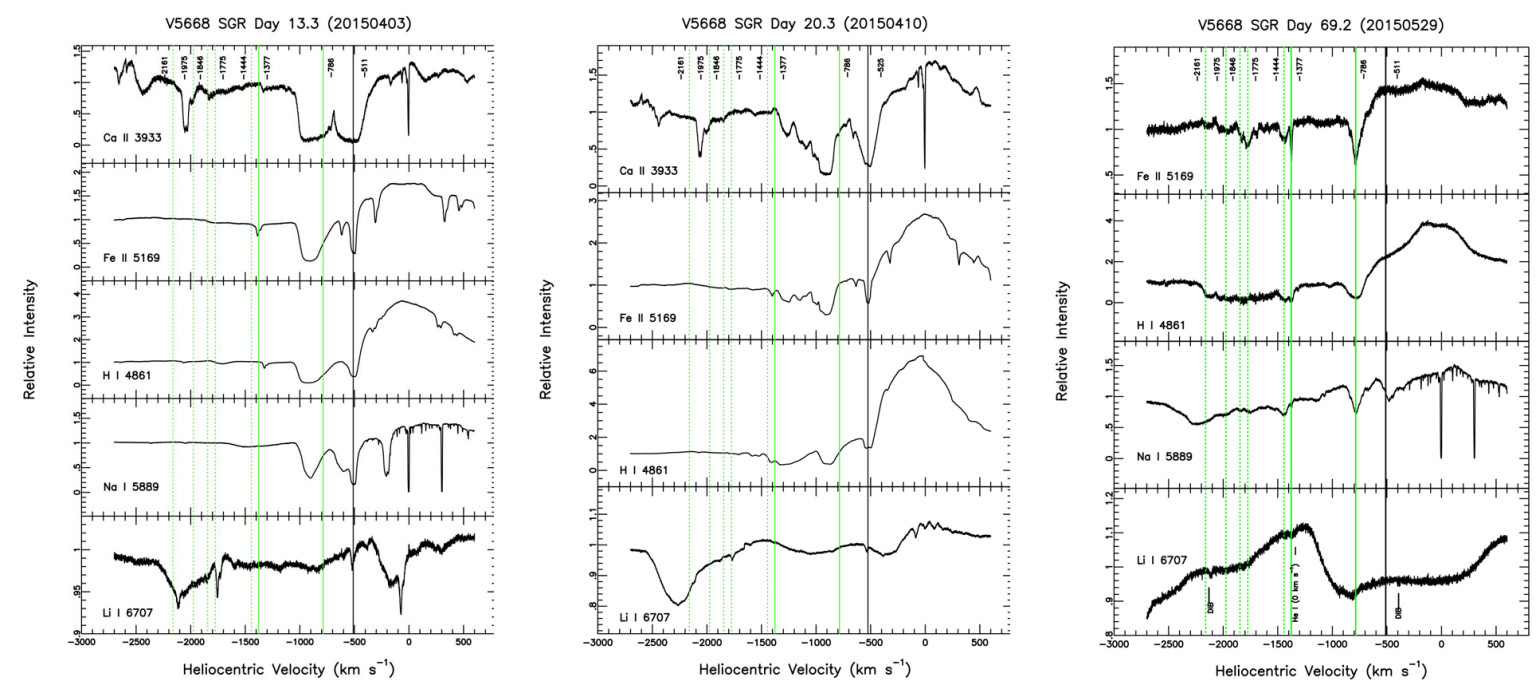

Figure 11: Spectra were obtained on 30 nights between 2015 April 3 and 2016 June 5. 12 epochs using either $1 \times 8.4 \mathrm{~m}$ or $2 \times 8.4 \mathrm{~m}$ LBT mirrors. 18 epochs using the $1.8 \mathrm{~m}$ VATT fiber feed so that our spectral resolution was either $270,000(1 \mathrm{~km} / \mathrm{s})$ or $120,000(2 \mathrm{~km} / \mathrm{s})$ for the LBT and a fixed 120,000 for the VATT. Dispersion $7 \mathrm{m \AA} /$ pixel. Our spectral coverage included all or part of the $3830 \AA$ to $9065 \AA$ region. These spectra were reduced using the PEPSI pipeline and are not smoothed. These 3 plots show V5669 Sgr on 3 days (day number is given on top of each panel) emphasizing the ${ }^{7} \mathrm{Li}$ region and showing that the wavelength agrees in velocity with $\mathrm{Ca}, \mathrm{Fe}, \mathrm{H}$, and $\mathrm{Na}$. The ${ }^{7} \mathrm{Li}$ absorption line has faded by day 20.3 and is gone by day 69.2 as the ionization steadily increased.

outburst. The WD is growing in mass. We have shown this both by following one outburst with NOVA and repeated outbursts with MESA.

4. The multi-dimensional studies show that there is sufficient mixing during the TNR to agree with observations of the ejecta abundances (84, and references therein).

5. This mixing occurs via convective entrainment (dredge-up of core gas into the accreted gas) during the TNR and does not affect the total amount of accreted material.

6. Simulations with $50 \%$ core matter plus $50 \%$ accreted matter reached higher peak temperatures and ejected more material moving at higher velocities than those with only $25 \%$ core and $75 \%$ accreted matter. The exceedingly high ejecta velocities do not agree with the observations. This result can likely be fixed by increasing the mass accretion rate to values above $2.0 \times 10^{-10} \mathrm{M}_{\odot} \mathrm{yr}^{-1}$. Such studies are in progress.

7. Our simulations confirm that $\mathrm{CO}$ novae are overproducing ${ }^{7} \mathrm{Be}$ which decays to ${ }^{7} \mathrm{Li}$ after we have ended our simulations. This result is in agreement with the observations of enriched ${ }^{7} \mathrm{Be}$ in $\mathrm{CN}$ explosions. 


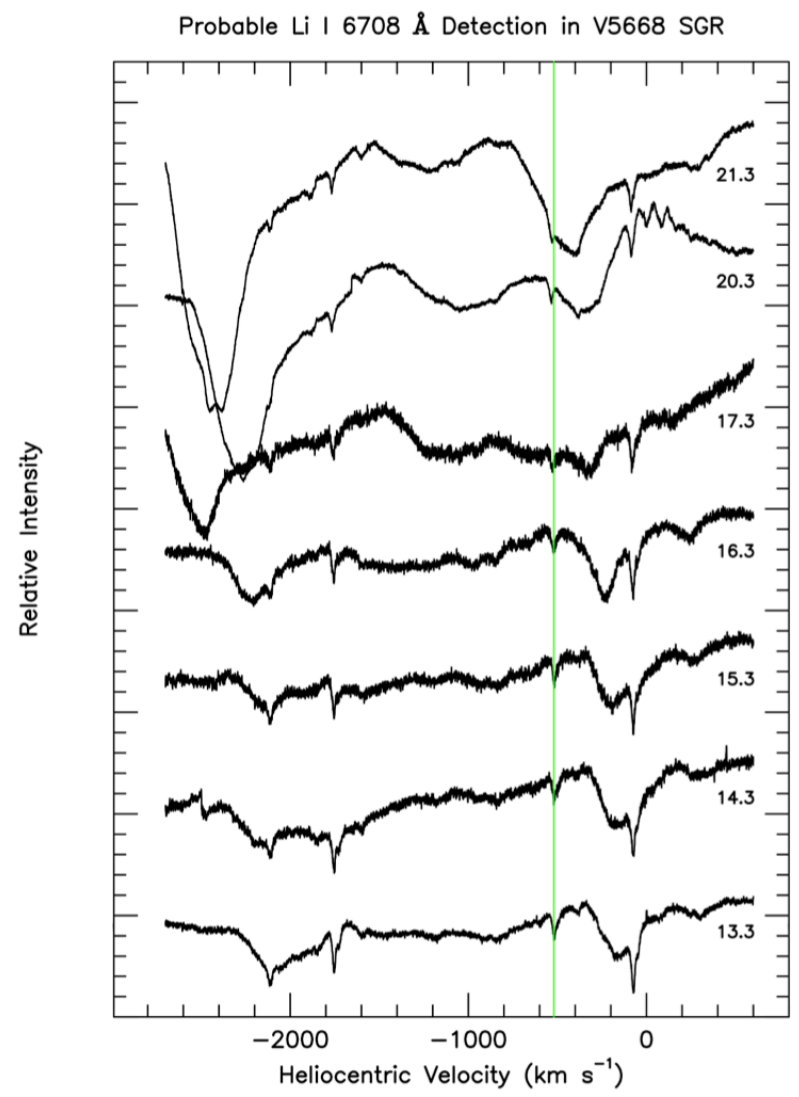

Figure 12: This figure shows the evolution of the lithium line from day 13.3 (our first observation) to day 21.3.

8. Simulations with $50 \%$ core and $50 \%$ accreted matter ejected, in some cases, a large fraction of the accreted material, implying that the white dwarf is shrinking in mass as a result of the nova.

9. Simulations with $25 \%$ core and $75 \%$ accreted matter ejected only a fraction of the accreted material implying that the white dwarf is growing in mass as a result of the Classical Nova phenomena. Since the properties of observed CO nova outbursts agree better with these simulations, we predict that the WD in the typical CO nova explosions is growing in mass as a result of the outburst.

\section{Acknowledgements}

SS thanks F. Giovanelli for inviting SS to give this talk and G. Shaviv for interesting discussions at the meeting. This work was supported in part by NASA under the Astrophysics Theory Program grant 14-ATP14-0007 and the U.S. DOE under Contract No. DE-FG02-97ER41041. SS acknowledges partial support from NASA and HST grants to ASU and WRH is supported by the U.S. Department of Energy, Office of Nuclear Physics. Our results benefitted from collabora- 
tions and/or information exchange within NASA's Nexus for Exoplanet System Science (NExSS) research coordination network sponsored by NASA's Science Mission Directorate. 


\section{References}

[1] B. Dilday, D. A. Howell, S. B. Cenko, J. M. Silverman, P. E. Nugent, M. Sullivan et al., PTF 11kx: A Type Ia Supernova with a Symbiotic Nova Progenitor, Science 337 (Aug., 2012) 942-, [1207.1306].

[2] M. L. Graham, C. E. Harris, O. D. Fox, P. E. Nugent, D. Kasen, J. M. Silverman et al., PTF11kx: A Type Ia Supernova with Hydrogen Emission Persisting after 3.5 Years, ApJ 843 (July, 2017) 102, [1706.02266].

[3] M. M. Phillips, The absolute magnitudes of Type IA supernovae, ApJ Let. 413 (Aug., 1993) L105-L108.

[4] A. V. Filippenko, Optical Spectra of Supernovae, ARAA 35 (1997) 309-355.

[5] D. A. Howell, Supernovae: A smashing success, Nature 463 (Jan., 2010) 35-36.

[6] B. Leibundgut, Type Ia Supernovae, Astr. \& Astrophys. Review 10 (2000) 179-209, [arXiv:astro-ph/0003326].

[7] B. Leibundgut, Cosmological Implications from Observations of Type Ia Supernovae, ARAA 39 (2001) 67-98.

[8] W. Hillebrandt and B. Leibundgut, eds., From twilight to highlight : the physics of supernovae : proceedings of the ESO/MPA/MPE workshop held at Garching, Germany, 29-31 July 2002, 2003. 10.1007/b80349.

[9] D. Maoz, F. Mannucci and G. Nelemans, Observational Clues to the Progenitors of Type Ia Supernovae, ARAA 52 (Aug., 2014) 107-170, [1312.0628].

[10] P. Ruiz-Lapuente, New approaches to SNe Ia progenitors, New Astronomy Reviews 62 (Oct., 2014) 15-31, [1403.4087].

[11] J. Whelan and I. J. Iben, Binaries and Supernovae of Type I, ApJ 186 (Dec., 1973) $1007-1014$.

[12] K. Nomoto, F.-K. Thielemann and K. Yokoi, Accreting white dwarf models of Type I supernovae. III - Carbon deflagration supernovae, ApJ 286 (Nov., 1984) 644-658.

[13] D. Branch, M. Livio, L. R. Yungelson, F. R. Boffi and E. Baron, In Search of the Progenitors of Type Ia Supernovae, PASP 107 (Nov., 1995) 1019-+.

[14] W. Hillebrandt and J. C. Niemeyer, Type IA Supernova Explosion Models, ARAA 38 (2000) 191-230, [arXiv:astro-ph/0006305].

[15] D. R. van Rossum, R. Kashyap, R. Fisher, R. T. Wollaeger, E. García-Berro, G. Aznar-Siguán et al., Light Curves and Spectra from a Thermonuclear Explosion of a White Dwarf Merger, ApJ 827 (Aug., 2016) 128, [1510. 04286$].$ 
[16] A. M. Khokhlov, Delayed detonation model for type IA supernovae, A\&A 245 (May, 1991) 114-128.

[17] D. Kasen, F. K. Röpke and S. E. Woosley, The diversity of type ia supernovae from broken symmetries, Nature 460 (08, 2009) 869-872.

[18] S. E. Woosley and D. Kasen, Sub-Chandrasekhar Mass Models for Supernovae, ApJ 734 (June, 2011) 38, [1010 . 5292].

[19] D. A. Howell, A. Conley, M. Della Valle, P. E. Nugent, S. Perlmutter, G. H. Marion et al., Type Ia supernova science 2010-2020, ArXiv e-prints: White Paper Submitted to the Astro 2010 Decadel Survey (Mar., 2009) , [0903.1086].

[20] K. Nomoto, T. Uenishi, C. Kobayashi, H. Umeda, T. Ohkubo, I. Hachisu et al., Type Ia Supernovae: Progenitors and Diversities, in From Twilight to Highlight: The Physics of Supernovae (W. Hillebrandt and B. Leibundgut, eds.), pp. 115-+, 2003.

[21] P. E. Nugent, M. Sullivan, S. B. Cenko, R. C. Thomas, D. Kasen, D. A. Howell et al., Supernova SN 2011 fe from an exploding carbon-oxygen white dwarf star, Nature 480 (Dec., 2011) 344-347, [1110.6201].

[22] W. Li, J. S. Bloom, P. Podsiadlowski, A. A. Miller, S. B. Cenko, S. W. Jha et al., Exclusion of a luminous red giant as a companion star to the progenitor of supernova SN $2011 \mathrm{fe}$, Nature 480 (Dec., 2011) 348-350, [1109.1593].

[23] J. S. Bloom, D. Kasen, K. J. Shen, P. E. Nugent, N. R. Butler, M. L. Graham et al., A Compact Degenerate Primary-star Progenitor of SN 2011fe, ApJ Let. 744 (Jan., 2012) L17, [1111.0966].

[24] L. Chomiuk, A. M. Soderberg, M. Moe, R. A. Chevalier, M. P. Rupen, C. Badenes et al., EVLA Observations Constrain the Environment and Progenitor System of Type Ia Supernova 2011fe, ApJ 750 (May, 2012) 164, [1201.0994].

[25] C. J. White, M. M. Kasliwal, P. E. Nugent, A. Gal-Yam, D. A. Howell, M. Sullivan et al., Slow-speed Supernovae from the Palomar Transient Factory: Two Channels, ApJ 799 (Jan., 2015) 52, [1405.7409].

[26] Y. Cao, S. R. Kulkarni, D. A. Howell, A. Gal-Yam, M. M. Kasliwal, S. Valenti et al., A strong ultraviolet pulse from a newborn type Ia supernova, Nature 521 (May, 2015) 328-331, [1505.05158].

[27] R. P. Olling, R. Mushotzky, E. J. Shaya, A. Rest, P. M. Garnavich, B. E. Tucker et al., No signature of ejecta interaction with a stellar companion in three type Ia supernovae, Nature 521 (May, 2015) 332-335.

[28] K. Maeda and Y. Terada, Progenitors of type Ia supernovae, International Journal of Modern Physics D 25 (July, 2016) 1630024, [1609.03639]. 
[29] R. M. Wagner, G. Schwarz and S. G. Starrfield, V445 Puppis, IAU Circulars 7571 (Jan., 2001) 1 .

[30] R. M. Wagner, G. Schwarz, S. G. Starrfield, C. B. Foltz, S. Howell and P. Szkody, V445 Puppis, IAU Circulars 7717 (Sept., 2001) 2.

[31] A. A. Henden, R. M. Wagner and S. G. Starrfield, V445 Puppis, IAU Circulars 7730 (Oct., 2001) 1.

[32] J. E. Lyke, C. E. Woodward, R. D. Gehrz, R. M. Wagner, S. G. Starrfield, G. Schwarz et al., V445 Puppis-An Unusual Nova?, in American Astronomical Society Meeting Abstracts \#198, vol. 33 of Bulletin of the American Astronomical Society, p. 803, May, 2001.

[33] P. A. Woudt and D. Steeghs, Spectroscopy and Near-Infrared Photometry of the Helium Nova V445 Puppis, in Interacting Binaries: Accretion, Evolution, and Outcomes (L. Burderi, L. A. Antonelli, F. D’Antona, T. di Salvo, G. L. Israel, L. Piersanti et al., eds.), vol. 797 of American Institute of Physics Conference Series, pp. 647-650, Oct., 2005, DOI.

[34] P. A. Woudt, D. Steeghs, M. Karovska, B. Warner, P. J. Groot, G. Nelemans et al., The Expanding Bipolar Shell of the Helium Nova V445 Puppis, ApJ 706 (Nov., 2009) 738-746, [0910.1069].

[35] T. Tomov, E. Swierczynski, M. Mikolajewski and K. Ilkiewicz, SALT observations of southern post-novae, A\&A 576 (Apr., 2015) A119, [1502.03462].

[36] M. Y. Fujimoto, A theory of hydrogen shell flashes on accreting white dwarfs. I - Their progress and the expansion of the envelope. II - The stable shell burning and the recurrence period of shell flashes, ApJ 257 (June, 1982) 752-779.

[37] M. Y. Fujimoto, A Theory of Hydrogen Shell Flashes on Accreting White Dwarfs - Part Two - the Stable Shell Burning and the Recurrence Period of Shell Flashes, ApJ 257 (June, 1982) 767.

[38] P. Kahabka and E. P. J. van den Heuvel, Luminous Supersoft X-Ray Sources, ARAA 35 (1997) 69-100.

[39] R. D. Gehrz, J. W. Truran, R. E. Williams and S. Starrfield, Nucleosynthesis in Classical Novae and Its Contribution to the Interstellar Medium, PASP 110 (Jan., 1998) 3-26.

[40] S. Starrfield, C. Iliadis, F. X. Timmes, W. R. Hix, W. D. Arnett, C. Meakin et al., Theoretical studies of accretion of matter onto white dwarfs and the single degenerate scenario for supernovae of Type Ia, Bulletin of the Astronomical Society of India 40 (Sept., 2012) 419, [1210.6086].

[41] E. P. J. van den Heuvel, D. Bhattacharya, K. Nomoto and S. A. Rappaport, Accreting white dwarf models for CAL 83, CAL 87 and other ultrasoft X-ray sources in the LMC, A\&A 262 (Aug., 1992) 97-105. 
[42] D. M. Townsley and L. Bildsten, Theoretical Modeling of the Thermal State of Accreting White Dwarfs Undergoing Classical Nova Cycles, ApJ 600 (Jan., 2004) 390-403, [arXiv:astro-ph/0306080].

[43] O. Yaron, D. Prialnik, M. M. Shara and A. Kovetz, An Extended Grid of Nova Models. II. The Parameter Space of Nova Outbursts, ApJ 623 (Apr., 2005) 398-410, [astro-ph/0503143].

[44] S. Starrfield, C. Illiadis and W. R. Hix, Thermonuclear Processes, in Classical Novae (M. F. Bode and A. Evans, eds.), pp. 77-101, Cambridge: University Press, 2008.

[45] G. S. Kutter and W. M. Sparks, Studies of Hydrodynamic Events in Stellar Evolution. I. Method of Computation, ApJ 175 (July, 1972) 407.

[46] W. M. Sparks and G. S. Kutter, Studies of Hydrodynamic Events in Stellar Evolution. II. Dynamic Instabilities in Stellar Envelopes, ApJ 175 (Aug., 1972) 707.

[47] G. S. Kutter and W. M. Sparks, Studies of Hydrodynamic Events in Stellar Evolution. III. Ejection of Planetary Nebulae, ApJ 192 (Sept., 1974) 447-456.

[48] G. S. Kutter and W. M. Sparks, Radial accretion of H-rich material onto a He white dwarf, ApJ 239 (Aug., 1980) 988-998.

[49] S. Starrfield, C. Iliadis, W. R. Hix, F. X. Timmes and W. M. Sparks, The Effects of the pep Nuclear Reaction and Other Improvements in the Nuclear Reaction Rate Library on Simulations of the Classical Nova Outburst, ApJ 692 (Feb., 2009) 1532-1542, [0811.0197].

[50] S. Starrfield, C. Iliadis and W. R. Hix, The Thermonuclear Runaway and the Classical Nova Outburst, PASP 128 (May, 2016) 051001.

[51] B. Paxton, L. Bildsten, A. Dotter, F. Herwig, P. Lesaffre and F. Timmes, Modules for Experiments in Stellar Astrophysics (MESA), ApJS 192 (Jan., 2011) 3-+, [1 009.1622 ].

[52] B. Paxton, M. Cantiello, P. Arras, L. Bildsten, E. F. Brown, A. Dotter et al., Modules for Experiments in Stellar Astrophysics (MESA): Planets, Oscillations, Rotation, and Massive Stars, ApJS 208 (Sept., 2013) 4, [1301.0319].

[53] B. Paxton, P. Marchant, J. Schwab, E. B. Bauer, L. Bildsten, M. Cantiello et al., Modules for Experiments in Stellar Astrophysics (MESA): Binaries, Pulsations, and Explosions, ApJS 220 (Sept., 2015) 15, [1506.03146].

[54] C. A. Iglesias and F. J. Rogers, Updated Opal Opacities, ApJ 464 (June, 1996) 943-+.

[55] A. L. Sallaska, C. Iliadis, A. E. Champagne, S. Goriely, S. Starrfield and F. X. Timmes, STARLIB: A Next-Generation Reaction-Rate Library for Nuclear Astrophysics, ApJS 207 (Apr., 2013) 18, [1304.7811]. 
[56] F. X. Timmes and D. Arnett, The Accuracy, Consistency, and Speed of Five Equations of State for Stellar Hydrodynamics, ApJS 125 (Nov., 1999) 277-294.

[57] F. X. Timmes and F. D. Swesty, The Accuracy, Consistency, and Speed of an Electron-Positron Equation of State Based on Table Interpolation of the Helmholtz Free Energy, ApJS 126 (Feb., 2000) 501-516.

[58] W. R. Hix and F.-K. Thielemann, Silicon Burning. II. Quasi-Equilibrium and Explosive Burning, ApJ 511 (Feb., 1999) 862-875, [arXiv:astro-ph/9808203].

[59] D. Arnett, C. Meakin and P. A. Young, Convection Theory and Sub-Photospheric Stratification, ApJ 710 (Feb., 2010) 1619-1626, [0910.0821].

[60] S. Cassisi, A. Y. Potekhin, A. Pietrinferni, M. Catelan and M. Salaris, Updated Electron-Conduction Opacities: The Impact on Low-Mass Stellar Models, ApJ 661 (June, 2007) 1094-1104, [arXiv:astro-ph/0703011].

[61] F. J. Rogers and A. Nayfonov, Updated and Expanded OPAL Equation-of-State Tables: Implications for Helioseismology, ApJ 576 (Sept., 2002) 1064-1074.

[62] D. Saumon, G. Chabrier and H. M. van Horn, An Equation of State for Low-Mass Stars and Giant Planets, ApJS 99 (Aug., 1995) 713-+.

[63] A. Y. Potekhin and G. Chabrier, Thermodynamic Functions of Dense Plasmas: Analytic Approximations for Astrophysical Applications, Contributions to Plasma Physics 50 (Jan., 2010) 82-87, [1001.0690].

[64] J. W. Ferguson, D. R. Alexander, F. Allard, T. Barman, J. G. Bodnarik, P. H. Hauschildt et al., Low-Temperature Opacities, ApJ 623 (Apr., 2005) 585-596, [astro-ph/0502045].

[65] M. Schwarzschild and R. Härm, Thermal Instability in Non-Degenerate Stars., ApJ 142 (Oct., 1965) 855.

[66] E. L. Schatzman, White dwarfs. Amsterdam, North-Holland Pub. Co.; New York, Interscience Publishers, 1958.

[67] J. N. Bahcall and R. M. May, The Rate of the Proton-Proton Reaction and Some Related Reactions, ApJ 155 (Feb., 1969) 501-+.

[68] M. Henze, M. J. Darnley, F. Kabashima, K. Nishiyama, K. Itagaki and X. Gao, A remarkable recurrent nova in $M$ 31: The 2010 eruption recovered and evidence of a six-month period, A\&A $\mathbf{5 8 2}$ (Oct., 2015) L8, [1508. 06205$].$

[69] M. J. Darnley, M. Henze, M. F. Bode, I. Hachisu, M. Hernanz, K. Hornoch et al., M31N 2008-12a - the remarkable recurrent nova in M31: Pan-Chromatic observations of the 2015 eruption, eprint ArXiv -1607.08082 (July, 2016), [1607.08082]. 
[70] N. J. Shaviv, Classical Novae as Super-Eddington Objects, in Classical Nova Explosions (M. Hernanz and J. José, eds.), vol. 637 of American Institute of Physics Conference Series, pp. 259-265, Nov., 2002, arXiv:astro-ph/0207639, DOI.

[71] S.-C. Yoon, N. Langer and M. van der Sluys, On the stability of thermonuclear shell sources in stars, A\&A 425 (Oct., 2004) 207-216, [arXiv: astro-ph/ 0406164 ].

[72] M. Zorotovic, M. R. Schreiber and B. T. Gänsicke, Post common envelope binaries from SDSS. XI. The white dwarf mass distributions of CVs and pre-CVs, A\&A 536 (Dec., 2011) A42, [1108.4600].

[73] J. Echevarría, E. de la Fuente and R. Costero, U Geminorum: A Test Case for Orbital Parameter Determination, AJ 134 (July, 2007) 262-273, [0 704 .1641].

[74] E. M. Sion, P. Godon, J. Myzcka and W. P. Blair, The Accreting White Dwarf in SS Cygni Revealed, ApJ Let. 716 (June, 2010) L157-L160, [10 07 . 3158].

[75] C. M. Copperwheat, T. R. Marsh, V. S. Dhillon, S. P. Littlefair, R. Hickman, B. T. Gänsicke et al., Physical properties of IP Pegasi: an eclipsing dwarf nova with an unusually cool white dwarf, Mon. Not. R. Astron. Soc. 402 (Mar., 2010) 1824-1840, [0911.1637].

[76] A. W. Shafter, On the masses of white dwarfs in cataclysmic binaries, Ph.D. thesis, California Univ., Los Angeles., 1983.

[77] S. Starrfield, R. D. Gehrz and J. W. Truran, Dust formation and nucleosynthesis in the nova outburst, in Astrophysical implications of the laboratory study of presolar materials (E. K. Zinner and T. J. Bernatowicz, eds.), vol. 402 of American Institute of Physics Conference Series, pp. 203-234, Mar., 1997, DOI.

[78] J. José, M. Hernanz, S. Amari, K. Lodders and E. Zinner, The Imprint of Nova Nucleosynthesis in Presolar Grains, ApJ 612 (Sept., 2004) 414-428, [arXiv:astro-ph/0405332].

[79] J. José, E. García-Berro, M. Hernanz and P. Gil-Pons, The First Nova Explosions, ApJ Let. 662 (June, 2007) L103-L106.

[80] N. Liu, L. R. Nittler, C. M. O’D. Alexander, J. Wang, M. Pignatari, J. José et al., Stellar Origins of Extremely 13C- and 15N-enriched Presolar SiC Grains: Novae or Supernovae?, ApJ 820 (Apr., 2016) 140, [1602.05252].

[81] J. José, G. M. Halabi and M. F. El Eid, Synthesis of C-rich dust in CO nova outbursts, A\&A 593 (Sept., 2016) A54.

[82] P. Haenecour, C. Floss, J. José, S. Amari, K. Lodders, M. Jadhav et al., Coordinated Analysis of Two Graphite Grains from the CO3.0 LAP 031117 Meteorite: First Identification of a CO Nova Graphite and a Presolar Iron Sulfide Subgrain, ApJ 825 (July, 2016) 88, [1606.08310]. 
[83] G. J. Schwarz, S. N. Shore, S. Starrfield, P. H. Hauschildt, M. Della Valle and E. Baron, Multiwavelength analyses of the extraordinary nova LMC 1991*, Mon. Not. R. Astron. Soc. 320 (Jan., 2001) 103-123.

[84] J. José, Multidimensional Modeling of Nova Outbursts, in Stella Novae: Past and Future Decades (P. A. Woudt and V. A. R. M. Ribeiro, eds.), vol. 490 of Astronomical Society of the Pacific Conference Series, p. 275, Dec., 2014.

[85] S. Starrfield, F. X. Timmes, C. Iliadis, W. R. Hix, W. D. Arnett, C. Meakin et al., Hydrodynamic Studies of the Evolution of Recurrent, Symbiotic and Dwarf Novae: the White Dwarf Components are Growing in Mass, Baltic Astronomy 21 (2012) 76-87, [1211.6145].

[86] G. Newsham, S. Starrfield and F. X. Timmes, Evolution of Accreting White Dwarfs: Some of Them Continue to Grow, in Stella Novae: Past and Future Decades (P. A. Woudt and V. A. R. M. Ribeiro, eds.), vol. 490 of Astronomical Society of the Pacific Conference Series, p. 287, Dec., 2014, 1303.3642.

[87] K. J. Kelly, C. Iliadis, L. Downen, J. José and A. Champagne, Nuclear Mixing Meters for Classical Novae, ApJ 777 (Nov., 2013) 130.

[88] A. G. W. Cameron and W. A. Fowler, Lithium and the s-PROCESS in Red-Giant Stars, ApJ 164 (Feb., 1971) 111.

[89] S. Starrfield, J. W. Truran, W. M. Sparks and M. Arnould, On Li-7 production in nova explosions, ApJ 222 (June, 1978) 600-603.

[90] M. Hernanz, J. Jose, A. Coc and J. Isern, On the Synthesis of 7Li and 7Be in Novae, ApJ Let. 465 (July, 1996) L27.

[91] F. X. Timmes, S. E. Woosley and T. A. Weaver, Galactic chemical evolution: Hydrogen through zinc, ApJS 98 (June, 1995) 617-658, [arXiv: astro-ph/9411003].

[92] L. Izzo, M. Della Valle, E. Mason, F. Matteucci, D. Romano, L. Pasquini et al., Early Optical Spectra of Nova V1369 Cen Show the Presence of Lithium, ApJ Let. 808 (July, 2015) L14, [1506.08048].

[93] K. G. Strassmeier, I. Ilyin, A. Järvinen, M. Weber, M. Woche, S. I. Barnes et al., PEPSI: The high-resolution échelle spectrograph and polarimeter for the Large Binocular Telescope, Astronomische Nachrichten 336 (May, 2015) 324, [1505.06492].

[94] A. Tajitsu, K. Sadakane, H. Naito, A. Arai, H. Kawakita and W. Aoki, The ${ }^{7}$ Be II Resonance Lines in Two Classical Novae V5668 Sgr and V2944 Oph, ApJ 818 (Feb., 2016) 191, [1601.05168].

[95] S. Starrfield, G. J. Schwarz, J. W. Truran and W. M. Sparks, The Effects of Metallicity on Hydrodynamic Studies of the Nova Outburst, in American Astronomical Society Meeting Abstracts \#194, vol. 31 of Bulletin of the American Astronomical Society, p. 977, May, 1999. 